\title{
UV STAR FORMATION RATES IN THE LOCAL UNIVERSE
}

\author{
Samir Salim, ${ }^{1,2}$ R. Michael Rich, ${ }^{1}$ Stéphane Charlot, ${ }^{3}$ Jarle Brinchmann, ${ }^{4}$ Benjamin D. Johnson, ${ }^{5}$ \\ David Schiminovich, ${ }^{5}$ Mark Seibert, ${ }^{6}$ Ryan Mallery, ${ }^{1}$ Timothy M. Heckman, ${ }^{7}$ Karl Forster, ${ }^{8}$ \\ Peter G. Friedman, ${ }^{8}$ D. Christopher Martin, ${ }^{8}$ Patrick Morrissey, ${ }^{8}$ Susan G. Neff, ${ }^{9}$ \\ Todd Small, ${ }^{8}$ Ted K. Wyder, ${ }^{8}$ Luciana Bianchi, ${ }^{10}$ José Donas, ${ }^{11}$ \\ Young-Wook Lee, ${ }^{12}$ Barry F. Madore, ${ }^{6}$ Bruno Milliard, ${ }^{11}$ \\ Alex S. Szalay, ${ }^{7}$ Barry Y. Welsh, ${ }^{13}$ and Sukyoung K. YI ${ }^{12}$ \\ Received 2006 December 22; accepted 2007 April 25
}

\begin{abstract}
We measure star formation rates (SFRs) of $\approx 50,000$ optically selected galaxies in the local universe $(z \approx 0.1)-$ from gas-rich dwarfs to massive ellipticals. We obtain dust-corrected SFRs by fitting the GALEX (ultraviolet) and SDSS photometry to a library of dust-attenuated population synthesis models. For star-forming galaxies, our UV-based SFRs compare remarkably well with those from SDSS-measured emission lines $(\mathrm{H} \alpha)$. Deviations from perfect agreement are shown to be due to differences in the dust attenuation estimates. In contrast to $\mathrm{H} \alpha$ measurements, UV provides reliable SFRs for galaxies with weak $\mathrm{H} \alpha$, and where $\mathrm{H} \alpha$ is contaminated with AGN emission (1/2 of the sample). Using fullSED SFRs, we calibrate a simple prescription that uses GALEX far- and near-UV magnitudes to produce dust-corrected SFRs for normal star-forming galaxies. The specific SFR is considered as a function of stellar mass for (1) star-forming galaxies with no AGNs, (2) those hosting an AGN, and (3) galaxies without $\mathrm{H} \alpha$ emission. We find that the three have distinct star formation histories, with AGNs lying intermediate between the star-forming and the quiescent galaxies. Star-forming galaxies without an AGN lie on a relatively narrow linear sequence. Remarkably, galaxies hosting a strong AGN appear to represent the massive continuation of this sequence. On the other hand, weak AGNs, while also massive, have lower SFRs, sometimes extending to the realm of quiescent galaxies. We propose an evolutionary sequence for massive galaxies that smoothly connects normal star-forming galaxies to quiescent galaxies via strong and weak AGNs. We confirm that some galaxies with no $\mathrm{H} \alpha$ show signs of star formation in the UV. We derive a cosmic star formation density at $z=0.1$ with significantly smaller total error than previous measurements.
\end{abstract}

Subject headings: galaxies: active — galaxies: evolution — galaxies: fundamental parameters — surveys — ultraviolet: galaxies

\section{INTRODUCTION}

Current studies of galaxies are characterized by two major features: availability of large samples of objects (galaxy surveys), and the utilization of the multiwavelength approach. Such studies extend from the nearby galaxies to those close to the epoch of the formation of the first galaxies. A rate at which a galaxy forms

\footnotetext{
1 Department of Physics and Astronomy, University of California, Los Angeles, CA 90095 .

2 Current address: National Optical Astronomy Observatory, 950 North Cherry Avenue, Tucson, AZ 85719; samir@noao.edu.

3 Institut d'Astrophysique de Paris, CNRS, 98 bis Boulevard Arago, F-75014 Paris, France.

4 Centro de Astrofísica da Universidade do Porto, Rua das Estrelas, 4150762 Porto, Portugal.

5 Department of Astronomy, Columbia University, New York, NY 10027.

${ }^{6}$ Observatories of the Carnegie Institution of Washington, 813 Santa Barbara Street, Pasadena, CA 91101.

7 Department of Physics and Astronomy, Johns Hopkins University, Homewood Campus, Baltimore, MD 21218.

8 California Institute of Technology, MC 405-47, 1200 East California Boulevard, Pasadena, CA 91125.

9 Laboratory for Astronomy and Solar Physics, NASA Goddard Space Flight Center, Greenbelt, MD 20771.

${ }_{10}$ Center for Astrophysical Sciences, Johns Hopkins University, 3400 North Charles Street, Baltimore, MD 21218.

${ }^{11}$ Laboratoire d'Astrophysique de Marseille, BP 8, Traverse du Siphon, 13376 Marseille Cedex 12, France.

12 Center for Space Astrophysics, Yonsei University, Seoul 120-749, Korea.

13 Space Sciences Laboratory, University of California, Berkeley, 601 Campbell Hall, Berkeley, CA 94720.
}

stars is one of the more important properties in studying galaxy evolution. The multiwavelength approach allows us to employ a suite of star formation (SF) indicators - from X-rays to radio wavelengths. Major effort in recent years was made to provide reliable calibrations for different SF estimators and to understand their differences, advantages and limitations. The most straightforward evaluation is achieved by comparing two or more star formation indicators for the same set of objects. While new galaxy surveys provide large statistical samples with which one can attempt such studies, the sample of galaxies for which more than a single star formation indicator can be applied is not necessarily large. Moreover, various SF indicators are often applied for different redshift regimes. Global cosmic star formation history is therefore the result of studies that employ different SF indicators.

Among the most frequently used star formation indicators are the UV continuum (usually at $\lambda<2000 \AA$ ), nebular recombination lines (primarily $\mathrm{H} \alpha$, but also [O II]), far-IR dust emission, and the synchrotron radio continuum at $21 \mathrm{~cm}$ (Kennicutt 1983). A comprehensive review of most of these methods was presented in Kennicutt (1998) together with simple formulae for the conversion of the true flux into a star formation rate (SFR). Recently, the use of other SF indicators has been explored, such as the X-ray continuum (e.g., David et al. 1992; Kilgard et al. 2002), or the luminosity of PAH features in the mid-IR (Roussel et al. 2001). Star formation indicators are only as good as the assumptions that connect a certain observed luminosity to the actual star formation rate, and therefore all are sensitive to various systematic uncertainties. One factor has proved more frustrating to account for than 
the others - the effect of dust obscuration on the UV and $\mathrm{H} \alpha$ star formation rates. In contrast to these two dust-sensitive indicators, bolometric IR luminosity and the radio luminosity are often considered to be "true" SF indicators. While this is certainly an oversimplification, such perception is bolstered by the very tight correlation of the IR and the radio luminosities, at least as observed for the normal galaxies in the local universe (de Jong et al. 1985). However, even if they were perfect, IR or radio methods cannot make UV or $\mathrm{H} \alpha$ methods obsolete for many practical reasons.

There have been a number of studies that compared the UV and $\mathrm{H} \alpha$ star formation rates-either as the observed luminosities, or by applying various schemes to correct for the dust attenuation. ${ }^{14}$ These comparisons were often carried out with respect to some dust-free SF indicator. Hopkins et al. (2001) used a sample of several hundred objects with $\mathrm{H} \alpha$ and UV measurements (with the $U$-band photometry serving as a proxy for the UV), and compared them to the far-IR measurements compiled by Cram et al. (1998). They find that in order to reconcile $\mathrm{H} \alpha$ and far-IR luminosities one needs to apply a dust attenuation that is not fixed, but rather depends on the SFR itself. Actually, this dependence is a consequence of the relationship between the dust attenuation and the far-IR luminosity found by Wang \& Heckman (1996). Hopkins et al. (2001) use an extinction curve to extrapolate the dust attenuations obtained for $\mathrm{H} \alpha$ into the UV regime, but find that such correction fails to bring UV luminosities to agree with the far-IR; i.e., the simple application of the same attenuation mechanism to both the $\mathrm{H} \alpha$ and the UV emission does not appear to be correct. Bell \& Kennicutt (2001) used actual UV observations of 50 nearby galaxies in two ultraviolet bands obtained with the Ultraviolet Imaging Telescope (UIT), and compared them to $\mathrm{H} \alpha$ luminosities from the narrowband imaging. They determine UV attenuation for 13 galaxies using the correlation with the UV slope (Calzetti et al. 1994), and measure $\mathrm{H} \alpha$ attenuation for 21 objects using the thermal radio continuum fluxes. They find that both can reach high levels even for normal galaxies ( $\sim 4 \mathrm{mag}$ for $\mathrm{H} \alpha$, and $\geq 5 \mathrm{mag}$ for far-UV), and lend support to previous notions that the attenuation increases with SFR. Sullivan et al. $(2000,2001)$ confirmed that a better agreement between the UV observations from the FOCA balloon mission (Milliard et al. 1992), and the fiber $\mathrm{H} \alpha$ spectra is achieved when attenuation corrections are taken to be luminositydependent. They also suggest that one perhaps cannot use simple extinction curve scalings to convert $\mathrm{H} \alpha$ attenuations into UV attenuations. The breakthrough in resolving this problem came with the introduction of the two-component dust attenuation model of Charlot \& Fall (2000). This model was indeed motivated by the need to produce a consistent model for dust attenuation affecting $\mathrm{H} \alpha$ and UV continuum photons. It postulates the existence of short-lived (10 Myr) giant molecular clouds that affect photons producing the $\mathrm{H} \alpha$ line. On the other hand, the attenuation of the UV continuum, having timescales longer than the lifetime of giant molecular clouds, is predominantly produced by the diffuse ISM (after the molecular clouds have dispersed), at levels that are typically 3 times lower (for a given wavelength) than those in the molecular clouds. In this paper we use the Charlot \& Fall (2000) model, thus testing it for the first time on a large scale.

In addition to systematic trends, we should mention that some previous studies were finding that the measurement errors are smaller than the observed scatter. Sullivan et al. (2000, 2001); Iglesias-Páramo et al. (2004) offer the explanation for this scatter

\footnotetext{
14 In this paper we use the term "attenuation," rather than the more commonly used term "extinction," thus emphasizing the complex processes of absorption and scattering in a galaxy, rather than the dimming of light along a single line of sight.
}

as arising from the differing timescales over which $\mathrm{H} \alpha$ and UV SFRs are sensitive, so that UV could in some cases (especially in low-mass galaxies) detect a short starburst that is no longer observable in $\mathrm{H} \alpha$.

Sometimes, the systematics are present in the observations themselves. Rosa-González et al. (2002) showed that even the relatively reliable determination of $\mathrm{H} \alpha$ attenuation can be affected by the systematics when not correcting for the underlying stellar absorption in Balmer emission lines. Systematics can also arise with galaxy samples selected at different wavelengths (Buat et al. 2002).

The main obstacle in obtaining UV measurements for a large number of galaxies in the local universe is that they need to be made outside of Earth's atmosphere. For this reason in 2003 NASA launched the Galaxy Evolution Explorer (GALEX; Martin et al. 2005). GALEX is currently conducting the first ever survey of the UV sky. The imaging (in two UV bands) is executed in several modes - from a shallow all-sky survey, to the ultradeep fields. In this paper we are using measurements obtained in the mediumdeep survey that is designed to image regions of the sky covered by the Sloan Digital Sky Survey (SDSS). Thus we obtain a large sample of galaxies with both UV and optical photometry, as well as spectroscopic redshifts. GALEX and SDSS data, and the resulting sample, are described in $\S \S 2$ and 3. Dust-corrected star formation rates (and some other physical properties such as the stellar mass) are obtained by comparing the observed colors to stellar population synthesis models to which the dust attenuation has been applied ( $(4)$. While we use full UV to optical SED, the SFRs are essentially constrained by the UV (Salim et al. 2005). The large size of our sample permits a robust statistical analysis. We compare our UV-based SFRs to the results of the major study of Brinchmann et al. (2004, hereafter B04), who use SDSS spectra to derive $\mathrm{H} \alpha$-based SFRs for $\sim 10^{5}$ galaxies ( $(5)$. They employ an intricate scheme to correct for the fiber aperture effects. Thus, our study also serves as a check on the reliability of their methodology. Finally, we discuss star formation histories of different classes of galaxies $(\S 7)$ and derive a UV-based estimate of the global star formation density at $z=0.1(\S 8)$.

\section{DATA}

\subsection{GALEX Data}

Technical aspects of GALEX telescope, detectors, and data products are presented in Morrissey et al. (2005, 2007). Here we give a summary of relevant information. GALEX surveys the sky in either the imaging or the grism spectroscopy mode. It simultaneously produces a far-UV (FUV) and a near-UV (NUV) image having a circular field of view of $1.25^{\circ}$ diameter. FUV and NUV filters are broadband, with effective wavelengths of 1528 and $2271 \AA$, respectively. We denote the magnitudes measured in these photometric bands as FUV and NUV. A single field of view imaged by GALEX is called a tile. A tile can consist of one or more "visits," i.e., individual exposures. GALEX surveys the sky in several imaging modes, which differ in the exposure time per tile. In this study we use the Medium Imaging Survey (MIS), which is designed to maximize the coverage of the sky that is included in the SDSS. Typical exposure times in MIS are $1500 \mathrm{~s}$, yielding limiting magnitudes of FUV $=\mathrm{NUV}=22.7 \mathrm{mag}$ ( $\mathrm{AB}$ system is used throughout). GALEX cannot point in the vicinity of bright sources (usually stars), which inevitably produces some gaps in the coverage.

GALEX data used in this paper come from the MIS portion of the Internal data release 1.1 (IR1.1), which is an expanded version of the first public GALEX release (GR1) of MIS (in both IR1.1 and 
GR1 the same pipeline, version 4, is used to reduce the data and produce source catalogs). The data set consists of 705 GALEX tiles. Because of the anomaly with the FUV detector, 98 tiles lack FUV images. The 705 tiles cover $797 \mathrm{deg}^{2}$ of the sky. Each GALEX field is restricted to $0.6^{\circ}$ radius, close to the maximum field of view. ${ }^{15}$ Because of the overlap between GALEX tiles, the total unique area is $741 \mathrm{deg}^{2}$. Source catalog for each tile was produced using the SExtractor software (Bertin \& Arnouts 1996).

In our study we use FUV and NUV fluxes measured in Kron elliptical apertures. We recalculate flux errors because they were incorrect in the pipeline version 4 reductions (thus also affecting GR1; e.g., Trammell et al. 2007). Kron magnitudes should represent a good measure of a galaxy's total flux. If both the FUV and the NUV detections are present, we use the aperture (size, shape, and position) derived from the NUV image to measure the FUV light. We go from NUV to FUV because we are generally more sensitive in the NUV. We add zero-point calibration errors of 0.052 and 0.026 mag to FUV and NUV, respectively, to account for systematic inaccuracies. The calibration errors were estimated by analyzing the repeat imaging of a calibration stellar object, and confirmed by a large number of repeat observations of the same field (Morrissey et al. 2007). If a UV detection is present in only one band, we still measure the formal flux and its error in the other band (using the aperture defined in the detected band). In some cases, the optical source is not detected at all by GALEX, in which case we note the sky background at the position of the SDSS source and compute the flux error. In this calculation we use galaxy sizes that have been derived (using a calibration based on objects detected by GALEX) from SDSS Petrosian radii in $r$ band. Finally, in some cases the FUV cannot be used because the FUV image does not exist (15\% of all galaxies).

\subsection{SDSS Data}

In addition to the ultraviolet data from GALEX, we use optical data from SDSS Data Release 4 (DR4; Adelman-McCarthy et al. 2006). SDSS is providing five-band broadband photometry (ugriz bands), and the spectroscopic follow-up of most galaxies with $r \leq 17.77$ (main galaxy spectroscopic survey; Strauss et al. 2002). In addition to the official data products from the SDSS collaboration, we use value-added galaxy catalogs produced by MPA/JHU SDSS team. These catalogs include reprocessed SDSS spectroscopic data, and some derived galaxy physical parameters that are based on the spectroscopic data.

SDSS catalog lists magnitudes measured in several different ways. We use MODELMAG magnitudes, which are the measurements of choice for relative fluxes (i.e., provide stable colors), while still capturing most of the total light. We apply slight adjustments ( -0.04 and $0.02 \mathrm{mag}$ ) to $u$ and $z$ magnitudes, respectively, to bring them closer to the actual $\mathrm{AB}$ system (Abazajian et al. 2004). We convert SDSS magnitudes and errors into fluxes using the transformations given in Scranton et al. (2005). In addition to catalog photometric errors, we add $0.01 \mathrm{mag}$ of uncorrelated calibration error to each of the bands (see $\S 4.3$ ), and, specifically for the $u$ band, we add an additional color-dependent error due to the red leak $\left[\sigma_{u, \mathrm{RL}}=0.0865(r-i)^{2}+0.0679(r-i)\right.$, based on Abazajian et al. 2004]. In rare cases an SDSS magnitude would be missing and such band has to be excluded from the analysis. We

\footnotetext{
${ }^{15}$ Many other studies with $G A L E X$ restrict analysis to $0.5^{\circ}$ or $0.55^{\circ}$ radius field of view. This is because artifacts are more common near the detector edge, and the PSF becomes distorted. Since in this study we match SDSS objects to GALEX sources, the chances of a match with an artifact are small. Also, while the PSF at the edges is distorted, the total GALEX flux is not affected (see also $\S 4.3$ ).
}

further exclude from analysis individual SDSS magnitudes that are nominally fainter than ugriz $=25$. These are invariably spurious, regardless of the listed photometry error. Finally, we exclude magnitudes with errors larger than 10 times the typical error in that band (we find typical errors to be $0.07,0.009,0.007,0.007$, and $0.017 \mathrm{mag}$ for $u, g, r, i$, and $z$ bands, respectively). We find that while large, these errors are significantly underestimated. To summarize, except in cases listed above when we exclude certain individual flux points, our input data consists of seven-band photometry and spectroscopic redshifts from SDSS.

\subsection{GALEX-SDSS Matched Catalog}

Of $741 \mathrm{deg}^{2}$. of GALEX unique imaging, $645 \mathrm{deg}^{2}$. overlaps with SDSS DR 4 spectroscopic area, thus defining the solid angle of our sample. ${ }^{16}$ We estimate this area by counting the SDSS galaxies that: (1) have spectra, (2) have a dereddened magnitude $14.5<r_{\text {Petro }}<17.5$ (the faint end is taken to be comfortably brighter than the spatially variable spectroscopic limit), and (3) lie at redshifts $0.005<z<0.22$. We count such galaxies both in our survey, and in the full DR4, whose spectroscopic area is known. The ratio of the two counts gives us the size of our survey area. This estimate should be good to within $1 \mathrm{deg}^{2}$. There are 67,883 objects from the SDSS DR4 spectroscopic survey (not restricted to the main galaxy survey) that lie in this area, and are spectroscopically classified as galaxies. For each of these objects we search for a match in the GALEX source catalog (which already combines FUV and NUV detections) within $4^{\prime \prime}$. Our analysis of SDSS point sources with a match in GALEX indicates that GALEX positions have a random error of $0.8^{\circ}$ in either R.A. or decl. (becoming somewhat larger at the edges of the field). In addition, there are overall tile-to-tile offsets between GALEX and SDSS coordinate systems of several tenths of an arcsecond. In any case, astrometric uncertainties are significantly smaller than our matching radius (see also Trammell et al. 2007). A genuine match can be missed if the centroid of the optical light is offset by more than $4^{\prime \prime}$ compared to the centroid of UV light. We expect such cases to be quite rare, since at the mean redshift of the sample $(z=0.104)$ this offset would translate into a $7 \mathrm{kpc}$ separation. A problem with matching in general is that what is considered to be a single object in one catalog can be resolved into multiple objects in another catalog, whether they are indeed separate objects (blending), or actually belong to the same system (shredding). This problem is more pronounced when combining catalogs with different resolutions, as is the case here $\left(4^{\prime \prime}-5^{\prime \prime}\right.$ for GALEX vs. $1.4^{\circ}$ for SDSS). However, in our particular sample this problem is not severe. SDSS galaxies with spectra are relatively bright objects, and if more than one GALEX object is found within the search radius our procedure was to take the one that is positionally the closest. Since we are dealing with high-latitude fields where the density of foreground stars is not that high, this simple matching procedure produces reliable results. Besides, we do have mechanisms of identifying the majority of incorrect matches at the later stage, when we perform an SED fitting to the combined GALEX-SDSS photometry (see $\S 4.3$ ). Since we combine photometry from two different catalogs there is a concern of photometric zero-point mismatch. We explore such a possibility in $\S 5.4$. In our final matched catalog we eliminate duplicate GALEX observations (stemming from overlaps or repeat observations) by keeping those that lies closer to the center of GALEX field of view.

\footnotetext{
${ }^{16}$ Matching with the current SDSS data release DR5 (which uses the same pipeline as DR4) would not significantly increase the overlap, since current GALEX pointings mostly follow the footprint of SDSS DR1 and DR2.
} 


\section{THE SAMPLE}

\subsection{Sample Selection}

We initially define our sample by applying magnitude and redshift cuts to galaxies with SDSS spectroscopy (note that objects spectroscopically classified as QSOs are excluded). We require the dereddened Petrosian magnitude to be in the $14.5<$ $r_{\text {Petro }} \leq 17.77$ range, where the faint limit is the nominal limit of the SDSS main galaxy spectroscopy survey (see also $\S 4.4$ ), while the bright limit is chosen to avoid objects with saturated SDSS photometry. We require redshifts to lie within $0.005<z \leq 0.22$ range. The lower limit is chosen to eliminate galaxies that may deviate from the Hubble flow, i.e., whose redshift distances could be unreliable. Redshift distribution in SDSS main galaxy sample peaks sharply around $z=0.1$ with few galaxies beyond our upper redshift limit. These magnitude and redshift limits are identical to those used in B04, to which we compare many of our results. We refer to the above redshift range as representing the "local universe."

There are 49,346 galaxies that meet the conditions defining our initial sample. Good quality SED fitting ( $\operatorname{see} \S 4.2$ ) is obtained for $98 \%$ of them (48,295 galaxies). In the rest of the paper we use only this latter sample and refer to it as "all" galaxies. Note that since we retain objects regardless of whether they were detected by $G A L E X$ (as long as they fall within the area covered by GALEX), our sample is only optically ( $r$-band) selected.

Throughout the paper we use $\Omega_{m}=0.3, \Omega_{\Lambda}=0.7, H_{0}=$ $70 \mathrm{~km} \mathrm{~s}^{-1} \mathrm{Mpc}^{-1}$ (i.e., $h_{70}=1$ ) cosmology.

\subsection{Emission-Line Diagnostics and Sample Classification}

This work focuses on the physical properties of galaxies, such as their star formation rates (SFRs) and stellar masses. Before we start analyzing the sample based on these derived properties, we would like to characterize it in terms of its observable quantities.

Throughout this paper we rely on optical emission lines to classify galaxies in our sample. This classification, based on the BPT diagram (Baldwin et al. 1981), plots the flux ratio of [O III] $\lambda 5007 \AA$ and $\mathrm{H} \beta$ lines against the flux ratio of $\left[\mathrm{N}_{\mathrm{II}}\right] \lambda 6584 \AA$ and $\mathrm{H} \alpha$ lines. In this paper we fully adopt the BPT classification of B04, which is based on emission-line strengths corrected for the underlying stellar absorption (see their Fig. 1). The position of a galaxy in the BPT diagram indicates the nature of its ionizing source. The majority of galaxies in the diagram fall within one of the two branches. One branch lies mostly above the maximum line ratios expected from star formation. These galaxies must have some ionizing source in addition to young stars. Most researchers attribute this emission to an active galactic nucleus (AGN). Specifically, the emission is associated with a narrow-line AGN (a LINER or a Seyfert 2), also known as a type 2 AGN (Kauffmann et al. 2003b). Note that any type 1 AGN spectrum (QSO or Seyfert 1) would have broad lines, and would be classified by the SDSS pipeline as a QSO and thus not included in this sample. Following Kauffmann et al. (2003c), B04 classify galaxies that lie on the bottom portion of the AGN branch, but with line ratios allowed by star formation, as "composite" (star-forming/AGN) galaxies. For galaxies that lie on the star-forming branch we expect very little emission-line flux to come from an AGN (Kauffmann et al. 2003b). B04 required a $\mathrm{S} / \mathrm{N}$ ratio in all four lines to be $>3$ in order to apply the BPT diagram classification. However, in some cases a secure AGN classification is possible even when only [N II] 6584 and $\mathrm{H} \alpha$ have $\mathrm{S} / \mathrm{N}>3$, i.e., in cases in which this ratio is larger than the one allowed by SF. B04 calls this class a "low-S/N AGN (LINER)." Following B04, we study this class together with the AGNs. B04 also introduce the category of "low-S/N SF" galaxies. These are the galaxies that do not fall in any of the previous categories because their lines have low $\mathrm{S} / \mathrm{N}$, yet they have an $\mathrm{H} \alpha$ detection with $\mathrm{S} / \mathrm{N}>2$. While their lines are not strong enough for secure placement on the BPT diagram, if we nevertheless do so, we find that many objects lie in the high-mass end part of the SF sequence, as well as in the bottom portion of the AGN branch. Therefore, these objects represent a heterogeneous class. Finally, there are galaxies without detectable lines, thus precluding the classification in the above scheme. B04 call this group "unclassifiable." We call them the "No $\mathrm{H} \alpha$ " class. We find that in $\sim 2 \%$ of galaxies in this class the $\mathrm{H} \alpha$ nondetection is due to some artifact in the spectrum or lineextraction pipeline. We exclude these galaxies from this class (but not from the whole sample). The fraction of galaxies in our sample belonging to different classes is as follows: Star-forming (SF), 27\%; Low S/N SF, 19\%; Composite (SF/AGN), 8\%; AGN, 12\%; and $\mathrm{No} \mathrm{H} \alpha, 33 \%$.

\subsection{UV-to-Optical Color-Magnitude Diagram}

Color-magnitude diagram (CMD) is a powerful tool in assessing the basic properties of a sample of galaxies. Historically, the study of CMDs was preceded by the studies of a relationship between color and morphology. Basic morphological segregation into disklike spiral galaxies and spheroidal elliptical galaxies was established by Hubble (1926). Afterward (e.g., Hubble 1936) it was realized that spiral (late-type) galaxies have bluer colors than the ellipticals (early types). Optical CMDs were constructed for cluster early type galaxies (e.g., Sandage 1972), where they were found to form a narrow sequence (the so-called red sequence). Field spiral galaxies also displayed the color-magnitude relationship (Chester \& Roberts 1964), albeit with larger scatter. The bimodal nature of the field galaxy CMD became much more apparent with recent large scale surveys, in particular SDSS (Baldry et al. 2004). However, unlike the traditional optical CMDs, a CMD in which the color is constructed from an ultraviolet and an optical magnitude has a particular diagnostic power. By virtue of contrasting the current (or recent) star formation as indicated by the UV light (modulo attenuation) to the total past star formation as indicated by the optical light, the UV to optical color is a good proxy of a galaxy's SF history (e.g., Salim et al. 2005). On the other axis, one plots absolute optical magnitude, which is related (modulo variations in the optical mass-to-light ratio) to galaxy's current stellar mass. We present the observed CMD of our sample in Figure 1. In this and many subsequent figures, the individual data points have been converted into a gray-scale density scatter plot, in such a way that the shade of gray is proportional to the number of objects occupying a given "pixel." Such representation is desirable when dealing with large samples where it is easy to saturate a traditional scatter plot. Unless noted otherwise, the figures are constructed from raw, unweighted counts. We include volume corrections below, when appropriate. Both the color and the absolute magnitude have been $K$-corrected to $z=0$ restframe bands ( $\S 4.4)$. The top left panel shows the CMD of all galaxies with an NUV detection ( $85 \%$ of the total sample; see Table 1 for a breakdown of UV detection rates per class). A striking feature of a UV to optical CMD is the pronounced bimodality of blue and red galaxies. Blue galaxies form a well-defined sequence extending to faint luminosities. The red sequence is somewhat more narrow than the blue sequence (note that this becomes evident only after $K$-corrections have been applied), and extends to intrinsically more luminous galaxies than the blue sequence. This is related to well-known fact that the most massive elliptical galaxies are more massive than the most massive spirals (Holmberg 


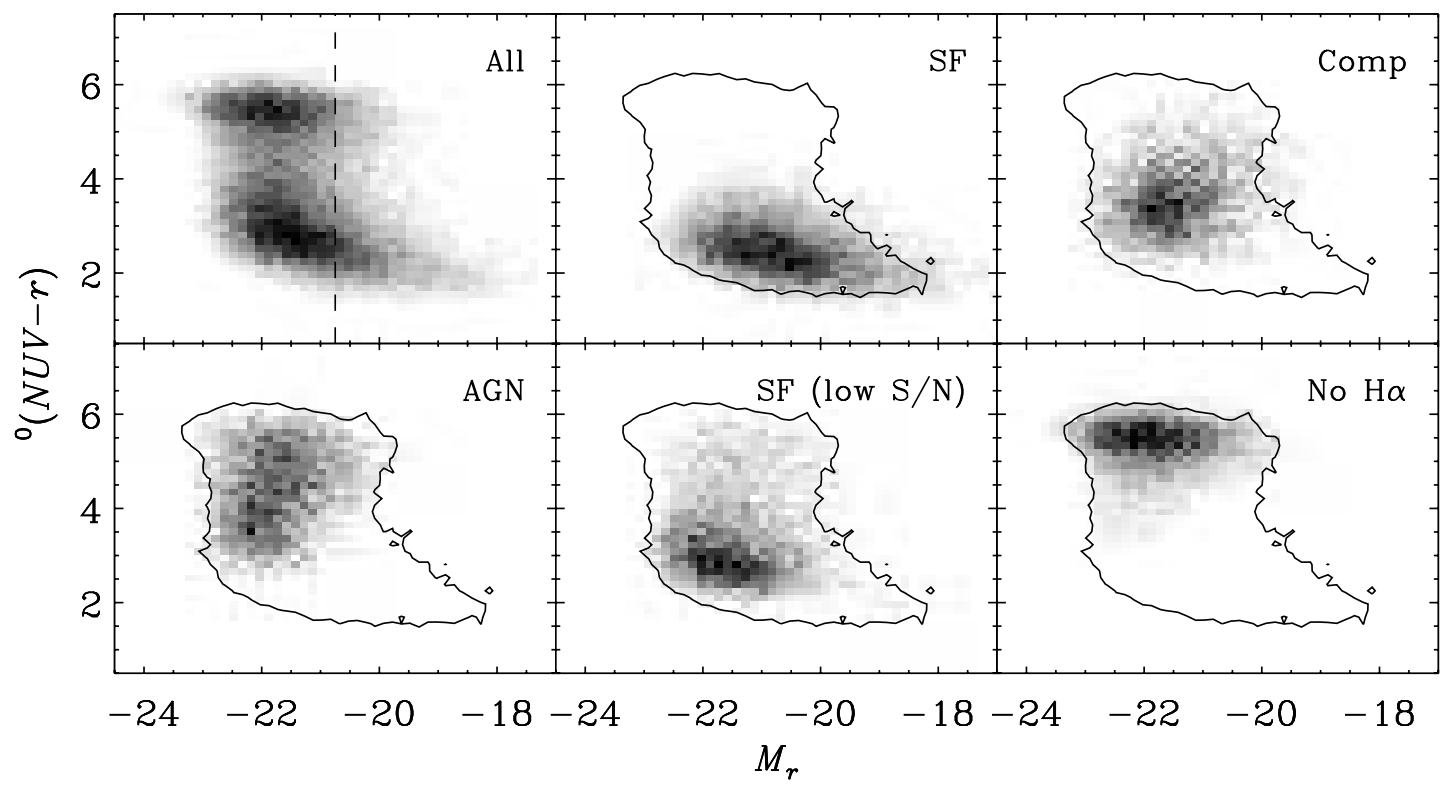

FIG. 1.-UV to optical color-magnitude diagrams (CMDs). Top left panel shows a gray-scale scatter plot of all galaxies in our sample with a near-UV detection. (The shade of gray is directly correlated to the number of points contained in a given "pixel.") Note the pronounced bimodality of the blue and the red sequences, and their large separation. Dashed line represents the completeness limit at the mean redshift of our sample. The remaining panels show CMDs of different galaxy classes (SF: starforming; Comp: star-forming/AGN composite; AGN: type $2 \mathrm{AGNs}$; $\mathrm{SF}$ ( low $\mathrm{S} / \mathrm{N}$ ): star forming with weak $\mathrm{H} \alpha$; and No $\mathrm{H} \alpha$ ), as determined from the position in the BPT diagram. The outer contour encompasses $90 \%$ of the entire sample is plotted for reference. While SF galaxies mostly lie in the blue sequence, and those with no H $\alpha$ in the red, most galaxies in between the two sequences are AGNs or AGN/SF composites. Absolute magnitude is given in $z=0 r$-band, and the color is $K$-corrected to $z=0$ rest-frame, as indicated by superscript 0 .

1965). The two sequences are separated by $\sim 3$ mag. If the two sequences are modeled as Gaussians, one finds that there is an excess of galaxies in the gap (Wyder et al. 2007). This is not the case in classical optical CMDs (Baldry et al. 2004). We refer to the gap region and its population as the "green valley" (Martin et al. 2007). A detailed quantitative study of the GALEX UV to optical CMD is presented in Wyder et al. (2007).

In the subsequent panels in Figure 1 we display the CMDs of various classes of galaxies as defined in $\S 3$ 3. Each panel has the gray scale normalized to the number of galaxies in the given class. For better reference with respect to the full sample, in each subsequent panel we repeat the contour containing $90 \%$ of all galaxies. Not surprisingly, the star-forming galaxies (SF) occupy the blue portion of the CMD. Part of the width of the sequence is due to the intrinsic dust attenuation. The CMD of composite galaxies (showing both signatures of SF and AGNs) are shown in the top right panel. Their NUV $-r$ colors are offset to the red compared to those of the "pure" SF galaxies. Galaxies with narrow line AGNs (bottom left panel) occupy the regions of the red sequence and of the green valley (Martin et al. 2007; Kauffmann et al.
2007). Moreover, most galaxies with intermediate colors are either AGNs or composites. Star formation histories of AGNs are discussed more extensively in $\S 7.3$. Next, we have low-S/N SF galaxies (middle bottom panel), which indeed mostly lie on the blue, star-forming sequence (preferentially its more luminous part). However, there is a tail of red galaxies that are probably contaminated by AGNs as observed in $\S 3.2$. Finally, we have galaxies with no detectable $\mathrm{H} \alpha$ (bottom right panel). As expected, these galaxies form the bulk of the red sequence. However, there is a tail of galaxies of this class extending toward the blue colors. Since the spectral classification used here is nuclear (central $\left.3^{\prime \prime}\right)$ it is possible that some of these galaxies are classified as not having $\mathrm{H} \alpha$ because of the dominant bulge, while the relatively faint star-forming disk is giving a galaxy an overall blue color. However, as we discuss in $\S 7.4$, this is not the case for most of them. The CMDs presented in Figure 1 require a detection in NUV. The remaining $15 \%$ of our sample with no NUV detection falls mostly in the red sequence (as evidenced from their $u-r$ colors). These galaxies are too faint to be detected in the UV.

TABLE 1

Average Errors of "UV" and "H $\alpha$ ”-Based Star Formation Rates

\begin{tabular}{|c|c|c|c|c|}
\hline Class & Number & $\begin{array}{l}\text { UV Detected }{ }^{\mathrm{a}} \\
(\%)\end{array}$ & $\left\langle\sigma\left(\log \mathrm{SFR}\left(“ U V^{\prime \prime}\right)\right)\right\rangle$ & $\langle\sigma(\log \mathrm{SFR}(“ \mathrm{H} \alpha ”))\rangle$ \\
\hline $\mathrm{All}^{\mathrm{b}}$ & 48,295 & 86 & 0.38 & 0.43 \\
\hline $\mathrm{SF}$ & 12,901 & 99 & 0.20 & 0.29 \\
\hline $\mathrm{SF}($ low $\mathrm{S} / \mathrm{N}) \ldots \ldots \ldots \ldots \ldots \ldots$ & 9060 & 93 & 0.30 & 0.39 \\
\hline Comp & 3966 & 96 & 0.28 & 0.40 \\
\hline AGN & 5827 & 90 & 0.41 & 0.49 \\
\hline No $\mathrm{H} \alpha$ & 16,159 & 68 & 0.60 & 0.54 \\
\hline
\end{tabular}

NOTE.-UV-based SFRs are averaged over $100 \mathrm{Myr}$.

${ }^{\text {a }}$ Either FUV or NUV detection. Of objects detected in FUV, 98\% are also detected in NUV.

${ }^{\mathrm{b}}$ Includes 382 objects for which classification was not possible; see $\S 3.2$. 


\section{OBTAINING GALAXY PROPERTIES WITH SED FITTING}

\subsection{SEDs of Model Galaxies}

Spectral energy distribution (SED) fitting is becoming a widely used technique for deriving galaxy properties. It was pioneered in works of Searle et al. (1973) and Larson \& Tinsley (1978). To first order it consists of comparing the observed SED to a set of model or template SEDs and searching for the best match. It is assumed that the physical properties of model or template galaxies are known, and that one can use this knowledge to deduce the properties of an observed galaxy.

In this study, we compare the observed SED to a large number of model SEDs constructed from the Bruzual \& Charlot (2003) population synthesis code. To construct the models we first choose input model properties by randomly selecting values from prior distributions defined in the following way. Formation of a galaxy is uniformly distributed between $0.1 \mathrm{Gyr}$ and the age of the universe. Global metallicity is uniformly distributed between 0.1 and $2 Z_{\odot}$. Star formation histories are not single stellar populations, but the combination of an exponentially declining continuous star formation, $\operatorname{SFR}(t) \propto \exp (-\gamma t)$, with $0 \leq \gamma \leq 1 \mathrm{Gyr}^{-1}$ uniformly distributed over that range, and the random starbursts superimposed on the continuous SF. Bursts are parameterized to have a duration uniformly distributed in the 30-300 Myr range, with a strength such that the mass produced in a burst is between 0.03 and 4 times the mass produced in the continuous SF over the present lifetime of a galaxy (distributed uniformly in log). Such parameterization conforms with most observational studies. Finally, bursts are randomly produced so that the probability that a given galaxy has undergone at least one burst over a 2 Gyr period is $50 \%$. The above parameters define how the population synthesis for each model will be carried out. Next, each of the above model SEDs is subjected to dust attenuation parameterized according to the two-component model of Charlot \& Fall (2000). For the $V$-band optical depth we randomly chose a value from a distribution $0<\tau_{V}<6$ (it peaks at $\tau_{V}=1.2$ and has most values in the $0<\tau_{V}<2$ range). The prior distribution for $\tau_{V}$ is empirical, and comes from Balmer decrements in SDSS spectra. The choice of $\tau_{V}$ prior distribution is discussed in $\S 5$.4. Fraction of the optical depth that affects stellar populations older than $10 \mathrm{Myr}$ (i.e., most of the UV continuum flux) is given by factor $\mu$, with values ranging from 0.1 to 1 , peaking around $\mu=0.3$. Altogether, we produce 100,000 model spectra spanning a range of SF histories, metallicities, and dust attenuations. Note that since we pick input parameters randomly, we do not call our set of models a grid, which would suggests a set of points with equal spacing in parameter space.

Finally, we convolve the resulting model spectra with $G A L E X$ and SDSS bandpasses at five redshifts equally spaced in the $[0.05$, 0.25 ] interval, producing the libraries of model broadband photometry. In each library we keep only models that have an age smaller than the age of the universe at that redshift. This effectively reduces the number of models from 95,000 at $z=0.05$ to 78,000 at $z=0.25$. We also add effects of the intervening intergalactic extinction according to Madau et al. (1996). Our final libraries list model photometry as well as a number of galaxy parameters associated to that model (such as the SFR averaged over several timescales, stellar masses, dust attenuation parameters).

\subsection{SED Fitting}

In our study, the observed SED is constructed from GALEX and SDSS broadband photometric fluxes (the broadband SED). For an observed galaxy at some redshift we select the model li- brary with the closest redshift. We step through a library one model at the time. Model flux points will have some arbitrary zero point, and in order to see how well an observed flux compares to the model flux, we first need to find a factor $a$ that minimizes the $\chi^{2}$ between the observed and the model points. In other words, for model $i$, we need to minimize the following expression:

$$
\chi_{i}^{2}=\sum_{X}\left[\frac{F_{\mathrm{obs}, X}-a_{i} F_{\bmod _{i}, X}}{\sigma\left(F_{\mathrm{obs}, X}\right)}\right]^{2} .
$$

Here, the summation is over $X=(\mathrm{FUV}, \mathrm{NUV}, u, g, r, i, z)$, the observed flux points are $F_{\text {obs }}$, and their errors $\sigma\left(F_{\text {obs }}\right)$. Flux points of model $i$ are $F_{\bmod _{i}}$. By taking the derivative of equation (1) with respect to $a$, and equating it with zero, we find the scale factor $a^{*}$ that best matches the observed and the model flux points:

$$
a_{i}^{*}=\sum_{X}\left[\frac{F_{\mathrm{obs} X} F_{\bmod _{i}, X}}{\sigma^{2}\left(F_{\mathrm{obs}, X}\right)}\right] / \sum_{X}\left[\frac{F_{\bmod _{i}, X}}{\sigma\left(F_{\mathrm{obs}, X}\right)}\right]^{2} .
$$

Because we include nondetections that have meaningless fluxes (small or even negative values), we use the observed fluxes without correcting for Galactic reddening, and instead apply the reddening to model fluxes, which are always positive. This allows us to treat nondetections like any other flux point with a known flux error. Reddening corrections for SDSS magnitudes come from the SDSS database, and those for GALEX from relations in Wyder et al. (2007; their eq. [2] for NUV).

The goodness of the fit between an observed galaxy and model $i$ is then obtained by inserting $a_{i}^{*}$ in place of $a_{i}$ in equation (1). Note that only a single parameter is being fit - the scale factor $a$. We emphasize that the word "parameter" when fitting is discussed should not be confused with the (in principle) arbitrary number of galaxy parameters (i.e., properties) that correspond to each model SED. Therefore, since we have seven photometric points, there are six degrees of freedom. For a given galaxy we evaluate $\chi_{i}^{2}$ for each model as outlined above. Now, each of these $\chi_{i}^{2}$ values will determine the relative weight $w_{i}$ for the given model $i$ (and therefore the weight of the galaxy parameters associated with that model) as $w_{i}=\exp \left(-\chi_{i}^{2} / 2\right)$. We then build a probability distribution function (PDF) for every galaxy parameter of interest by compounding weights at the corresponding parameter value. We repeat the procedure for each of the $\sim 10^{5}$ models in the given redshift library. We normalize the final PDFs and note the parameter values corresponding to the $2.5,16,50$ (median), 84 , and 97.5 percentiles of the cumulative PDF. We use the average of the PDF as our nominal estimate of the parameter value. Since in the general case the PDF is not symmetric, medians, averages, and modes will differ. For most relevant parameters in this study the differences between the median and the average are small. We do not consider using a mode, because due to the discreteness of the model parameter space, the mode can be stochastically offset from the bulk of the PDF and is sensitive to PDF binning. For similar reasons we do not use a single model with the best $\chi^{2}$ (i.e., the maximum likelihood model) as representative of parameter values, but we do keep track of the best $\chi^{2}$ values in order to evaluate the overall quality of the fitting for a given galaxy $(\S 4.3)$. In addition, for certain parameters we preserve more detailed information on the shape of the PDF (in the case of the specific SFR and stellar mass, we actually keep their mutual two dimensional PDFs). In certain cases we discuss the errors of the derived parameters. We use $1 / 4$ of the $2.5-97.5$ percentile range as a proxy for what would have been a $1 \sigma$ error in the Gaussian distribution. We refer to these quantities as "formal" errors. 
We prefer the above-described Bayesian approach (e.g., Leonard \& Hsu 2003) to often-used maximum likelihood, since it allows one to transparently test the dependence on the prior, i.e., how well the data constrain certain parameters given that the shape of the resulting PDF can to some extent depend on the distribution of the given parameter in the model library (especially when the observational constraints are poor). Another advantage is that while the maximum-likelihood model might indeed correspond to a high probability density, in the case of extensive models such as ours, it might actually correspond to a negligible probability "mass," and might therefore lead to misleading parameter estimation (see for instance the discussion in MacKay 2003). Note also that our technique properly accounts for the degeneracies inherent in some galaxy properties. Imagine that we have two galaxy parameters whose values can be picked in such a way as to produce identical model SEDs. In such a case our fitting will give equal probabilities to all of the degenerate models, and the resulting PDFs for each of these two parameters will therefore reflect the entire range that these parameters can take (i.e., there will be nothing to constrain the PDFs). If we then characterize the errors of those parameters using the width of the PDF, we will obtain very large values; i.e., we will know that a certain parameter is poorly constrained. This, in effect, is in contrast to fitting using a single best-fit model, which would pick one of the degenerate models as the best-fitting (and the corresponding parameter value), without giving a researcher an idea that there is an underlying degeneracy making the obtained value to be quite uncertain. Finally, we want to emphasize that the widths of the PDFs (and also their typical values) have a meaning only if the observational errors have been estimated reasonably correctly, and if the overall fit (which can be well characterized by the $\chi^{2}$ of the best-fitting model) is reasonably good $(\S 4.3)$.

\subsection{Quality of a Fit}

For our initial sample of 49,346 galaxies we obtain model parameters in the way described in $\S 4$.2. For each galaxy we also note the $\chi^{2}$ of the single best-fitting model, $\chi_{\text {best }}^{2}$. This value serves as an indicator of the overall quality of the fitting for a given galaxy. Since we include galaxies close to the edge of GALEX field of view where we know that image PSFs get distorted, we would first like to check if this affects the photometry, and therefore the quality of SED fitting. Thus, we look at the average $\chi_{\text {best }}^{2}$ as a function of distance from the GALEX image center. We find no correlation with the distance or degradation close to the edge.

In the ideal case, $\chi_{\text {best }}^{2}$ values would form a theoretical $\chi^{2}$ distribution. The average of such distribution should equal the degrees of freedom (in our case 6). Before looking at the $\chi^{2}$ distribution we notice that $\chi_{\text {best }}^{2}$ values are systematically higher for blue galaxies compared to the red. We believe that this difference arises from the presence of emission lines in blue galaxies that effectively increase the discrepancy between the observed SED and the model (line-free) continuum. Therefore, we start by comparing the observed and the theoretical $\chi^{2}$ distribution of red galaxies. Initially, the observed distribution was shifted toward the values that were too low. This was indicative of photometry errors being overestimated. We arrive at the $\chi^{2}$ distribution that was wellmatched to the theoretical one when we adopt 0.01 mag calibration errors in each of the five SDSS bands. ${ }^{17}$ While now the bulk

\footnotetext{
17 The "official" SDSS calibration errors are given as $2 \%$ for $r$ band, and between $2 \%$ and $3 \%$ for each of the four colors. However, the individual magnitude errors (which figure in eq. [1]) are correlated, which is why we find them to be smaller than $2 \%$. Our results stay practically the same even if errors are increased, but the $\chi^{2}$ distribution then deviates from the expected one.
}

of the red galaxies follows a $\chi^{2}$-like distribution, there is also present a tail of high $\chi_{\text {best }}^{2}$ values, in numbers well above any expected distribution. One can imagine that the main reason for the presence of $\chi^{2}$ outliers is that the data do not reflect the actual SED for some reason. We will investigate possible causes momentarily. The high- $\chi^{2}$ tail of red galaxies contains some $2 \%$ of objects. We decide to exclude these galaxies from further analysis (although we do account for them statistically when making any kind of space density calculation). Assuming that the reasons driving extreme $\chi^{2}$ values are color independent, we also clip $2 \%$ of the blue-galaxy $\chi_{\text {best }}^{2}$ distribution tail. This leaves us with 48,295 galaxies - our final sample.

While removing the outliers is necessary to have results not be biased by unreliable values, it is instructive to understand what causes some $\chi^{2}$ to assume such high values. We thus examine 22 galaxies with extreme $\chi_{\text {best }}^{2}$ values, distributed evenly in the $-1<\mathrm{NUV}-r<7$ range (thus sampling galaxies with various $\mathrm{SF}$ histories). In 10 cases we find obvious problems with cataloged photometry. This is most often the case with SDSS (nine objects), where the visual inspection of broadband SEDs and comparison with spectra indicates that some flux points are large outliers. Next, in seven cases we are dealing with either close galaxies being blended in GALEX (4), or with SDSS shredding galaxies into smaller photometric objects (2). For one object it is unclear whether we have blending or shredding. Further, in one case an object shows a clear QSO spectrum; i.e., the continuum is affected by the AGNs, yet it is spectroscopically (mis)classified as a galaxy. Finally, in four cases it appears that the broadband SEDs (and spectra) are genuinely unusual. While their number is too small to affect the present study, this type of outliers deserves a special scrutiny. It needs to be explored if there exists any set of model input parameters (SFH, dust attenuation, metallicity) beyond our already wide range, which can produce a model SED matching these observations. For our full sample we also expect on the order of 10 cases in which a foreground star of similar brightness is superimposed with a galaxy, and falls within the fiber, thus producing a "composite" galaxy/star spectrum that would fail the fitting.

Since our model libraries are calculated at some fixed redshifts, there is some error associated with the library redshift not perfectly matching the galaxy redshift. We thus contrast the $\chi_{\text {best }}^{2}$ values of galaxies having the maximum redshift offset (close to 0.025 ) to those with no offset. We find a systematic increase in $\chi_{\text {best }}^{2}$ of $10 \%$, which is too low to affect the overall $\chi^{2}$ distribution.

Finally, we want to assess the effects of the "resolution" of our model parameters; i.e., we can ask how extensive should a model library be that the resolution is not an issue. To that extent, we artificially degrade our model libraries by running full SED fitting using every second, every fourth and every eighth model SED. We then compare average $\chi_{\text {best }}^{2}$ values from each run. Overall, the quality of the fits are surprisingly stable. The run with every second model produces only a $4 \%$ increase in $\chi_{\text {best }}^{2}$ values, while the run using every 16 th model is $16 \%$ worse than with the full library. This implies that increasing the extent of the library (for the given range of input parameters) would not bring significant improvements. This, of course, is not a general conclusion, but rather states that with the precision of our data the current number of models is adequate; i.e., having a finer resolution of input model parameters would create model SEDs that are degenerate from the observational point of view. In addition to this, the Bruzual \& Charlot (2003) models themselves are not likely to be of such an accuracy to warrant more extensive model library. 


\subsection{K-Corrections and Volume Corrections}

For deriving the galaxy properties from the SED fitting, we are not required to know the $K$-corrections. This is because for a galaxy at a given redshift we compare the observed magnitudes to model magnitudes with bandpasses that have been shifted to the matching galaxy's redshift (i.e., the redshift of the model library). Therefore, the " $K$-corrections" are present in the SED fitting implicitly. However, for some applications, such as constructing a CMD ( $\S 3.3)$ or obtaining $V_{\max }$ weights ( $\S \S 2$ and 3$)$, we need to know the $K$-corrections explicitly. The process of SED modeling allows us in principle to derive the $K$-corrections alongside galaxy parameters. It even has the advantage of allowing one to construct a PDF for any $K$-corrected magnitude, which thus yields an estimate of a $K$-correction error. We find this approach to be especially useful in $K$-correcting higher redshift $(z \sim 1)$ samples (Salim et al. 2008, in preparation). In the current case, given that our redshift range is covered by only five model libraries, we choose to calculate the $K$-corrections using the publicly available code KCORRECT v4_1_4 ${ }^{18}$ (Blanton \& Roweis 2007). The code allows GALEX magnitudes to be used together with SDSS magnitudes to constrain the SED fit from which $K$-corrections are derived. We obtain $K$-corrections using the same combined GALEX-SDSS photometry used for the SED fitting, except that $<3 \sigma$ UV detections are not used. We derive $K$-corrections without a priori assuming an evolution of the luminosity function. We explicitly correct for evolution where appropriate.

In cases for which we require a volume-corrected sample, we assign a weight to each galaxy according to its $V_{\max }$ value - the volume in which a galaxy would be visible taking into account redshift and apparent magnitude limits, and the solid angle of a survey. While our faint limit $(r=17.77)$ is also the nominal limit of the SDSS main spectroscopic survey, we take into account that the actual spectroscopic magnitude limit varies from one spectroscopic plate to another (i.e., that it can be brighter than $r=17.77$ ). Thus, for each object we modify the volume by the spectroscopic completeness (usually around 0.9). We take spectroscopic limits and completeness values from NYU-VAGC catalog (Blanton et al. 2005). We caution that in order to calculate $V_{\max }$ correctly, it has to be done iteratively, because the $K$-correction depends on redshift limits that determine $V_{\max }$.

\section{COMPARISON OF "UV" AND "H $\alpha$ " STAR FORMATION RATES}

\section{1. “UV” SFR Estimates}

We derive our SFRs from photometric constraints that extend from the UV to the $z$ band. However, it is the UV luminosity that constrains the SFR the most. Therefore, in the remainder of the paper we refer to SFRs derived from the SED fitting as "UV," where quotation marks stand to remind us that it is not only UV information that contributed to these SFR estimates. In our model SED libraries we report "current" star formation rates averaged over several time intervals, most notably over the most recent $10 \mathrm{Myr}, 100 \mathrm{Myr}$, and 1 Gyr. By comparing the formal errors of SFR estimates (derived from the 95th percentile range of each galaxy's SFR PDF), we find that the best constrained SFR for the overall sample is the one averaged over the last $100 \mathrm{Myr}$ (Table 1), which is the timescale for UV-bright stars. For the starforming class specifically, we find that the SFRs have slightly smaller formal errors over 1 Gyr timescales than over 100 Myr. On the other hand, SFR estimates on timescales of $10 \mathrm{Myr}$ for SF galaxies (a timescale that matches $\mathrm{H} \alpha \mathrm{SFR}$ ) have drastically larger

18 Available from http://cosmo.nyu.edu/blanton/kcorrect. formal errors, as expected since we do not have information to constrain them. In the remainder of the paper we use SFR estimates averaged over $100 \mathrm{Myr}$, which represent a good compromise between the quality and a timescale that is not too long compared to that of the $\mathrm{H} \alpha$ SFRs.

\section{2. " $\mathrm{H} \alpha$ ” SFR Estimates}

B04 have developed a method of deriving SFRs from SDSS spectra that is primarily based on the intensity of an $\mathrm{H} \alpha$ line. This represents the largest currently available sample of precise $\mathrm{H} \alpha$-based SFRs. Obtaining accurate SFRs from SDSS spectra is far from straightforward. For the purposes of this paper we briefly describe the procedures employed in B04. The essence of the B04 approach was to model SDSS spectra by first removing the absorption line spectrum using a combination of burst models from Bruzual \& Charlot (2003) and then to model the emission lines using the Charlot \& Longhetti (2001) models that combine the Bruzual \& Charlot (1993) galaxy evolution models with the Cloudy photoionization code (Ferland 1996). Also, the same twocomponent dust attenuation prescription of Charlot \& Fall (2000) was used by B04 to model attenuation of the emission lines, and by us to model broadband continuum attenuation. While conventionally the attenuation of $\mathrm{H} \alpha$ flux is determined by comparing the observed $\mathrm{H} \alpha$ to $\mathrm{H} \beta$ ratio (Balmer decrement) to the theoretical one, the attenuation estimate in B04 is constrained using many emission lines (although it is dominated by $\mathrm{H} \alpha / \mathrm{H} \beta$ ). Using a suite of models, B04 apply a Bayesian approach to produce the probability distributions for each of the four parameters (gas metallicity, ionization parameter, dust attenuation, and dust-to-metal ratio). B04 thus simultaneously produce attenuation estimate as well as the attenuation-corrected SFR within the SDSS $3^{\prime \prime}$ fiber. SFR within the fiber is constrained by many emission lines, with the greatest weight carried by $\mathrm{H} \alpha$. This is one reason why we denote B04 SFR and attenuation estimates by " $\mathrm{H} \alpha$," with quotation marks again serving to indicate that not only $\mathrm{H} \alpha$ was involved in those estimates. The above procedure is directly applicable only to galaxies for which the ionizing source is predominantly star formation, and not, for example, AGN activity. B04 therefore calculate fiber SFRs directly from emission lines only for galaxies classified in the BPT diagram as star-forming and low-S/N starforming ( $\S 3.2$ ). For other classes of galaxies (AGNs, composite, galaxies with no $\mathrm{H} \alpha$ ) for which either there is no emission line detection or the lines are contaminated by a non-SF ionizing source, B04 use the relation between the D4000 spectral index and the specific SFR (SFR normalized by stellar mass) that has been calibrated using the star-forming galaxies. Therefore, for these galaxies, emission-line SFRs are used indirectly. This is another reason why we denote B04 SFRs as "H $\alpha$ " with quotation marks. We return to this calibration in $\S 5.3$. The above procedure gives only the SFRs within the $3^{\prime \prime}$ aperture of SDSS spectroscopic fibers, i.e., the fiber SFRs. In order to obtain total SFRs, an aperture correction is required. The total SFR is the sum of the SFR within the fiber and outside of it. To estimate the SFR outside of the fiber, galaxies are first divided into a color-color grid, based on their fiber colors. Then, for each color cell, a distribution of $i$-band luminosity normalized SFRs $\left(\mathrm{SFR} / L_{i}\right)$ is constructed by adding up all galaxies of $\mathrm{SF}$ or low-S/N SF class that fall within the given color cell. Then, the color of a galaxy outside of fiber is used to select the appropriate color cell, and the SFR $/ L_{i}$ in that cell is used with the $i$-band luminosity to derive the SFR outside of the fiber. This is added to previously found SFR within the fiber to obtain the total SFR. In other words, the aperture correction procedure is based on two assumptions. First, that SFRs within fibers have the same dependency on color as the SFR outside of it, and second, that this 
dependency, calibrated with star-forming galaxies, applies to other galaxy classes. The aperture correction factors range from close to one (no correction) to around a hundred fold. On average they are 0.9 dex for the entire sample and 0.6 dex for the star-forming class.

\subsection{Comparison of " $U V$ " and " $\mathrm{H} \alpha$ " SFRs for All Galaxies}

The comparison between SF indicators serves the obvious purpose of providing a better understanding of each indicator, but also ensures the mutual check on the reliability of the techniques used to produce the SFR estimates - in this case, the SFRs based on UV (this study) and on $\mathrm{H} \alpha$ (from B04).

We begin by comparing the formal errors of the two methods. Note that B04 used a sample based on the expanded SDSS DR1. Here, when we refer to B04 SFRs we mean the B04 calculations applied to SDSS DR4 and available as a part of MPA/JHU SDSS value-added catalog. In Table 1 we show average formal errors in SFR for the entire sample and for each galaxy class. We see that the formal errors of the two SF estimates happen to be comparable.

To facilitate the comparison, we first convert B04 SFRs that were calculated for Kroupa (2001) IMF to Chabrier (2003) IMF used in this study (both with $0.1-100 M_{\odot}$ limits). From Bruzual \& Charlot (2003) models we find the conversion factor of 1.06 (in the sense that Kroupa IMF SFRs are slightly higher). In Figure 2 we plot B04 "H $\alpha$ " SFR estimates against the "UV" for all galaxies in the sample (throughout the paper SFR always means dust-corrected SFR). Note that the "UV" SFRs exhibit 5 orders of magnitude of a dynamic range - from all but negligible rates to those approaching $100 M_{\odot} \mathrm{yr}^{-1}$. We notice that a large number of galaxies (those with higher "UV" SFRs) has comparable SFRs. However, there is a plume of galaxies for which B04 SFRs are up to 2 orders of magnitude higher than the "UV" ones. This systematic discrepancy cannot be explained by random errors of the two methods. Recall that B04 do not derive SFRs directly for classes other than star forming (and low $\mathrm{S} / \mathrm{N}$ star forming), but instead use relations calibrated on SF class sample. Thus, in Figure 3 we break the comparison into galaxy classes (omitting for the moment the SF class). For the low-S/N star-forming class (top left panel), for which B04 SFR estimates do come from the emission lines, the comparison is generally good for the majority of galaxies (apart from the overall offset that makes "H $\alpha$ " SFRs higher). For the other three classes in Figure 3, B04 SFRs rely on calibrations made using the SF galaxies. Composite (SF/AGN) galaxies compare relatively well, but the comparison becomes significantly worse for AGNs, where we can see both the significant offset for galaxies with higher SFRs, and the prominent plume for those with lower "UV" SFRs. Finally, for galaxies for which no $\mathrm{H} \alpha$ detections were possible, the discrepancy is very large for almost all galaxies. It is this class that contributes the most to the plume seen in the comparison of all galaxies (Fig. 2). Given that the galaxies without $\mathrm{H} \alpha$ detections belong almost exclusively in the red sequence (Fig. 1), the low SFRs rates estimated by the SED fitting ("UV") appear more realistic (see also $\S 7.1$ ). Also, such high SFRs as estimated by B04 would result in a high fraction of No $\mathrm{H} \alpha$ galaxies having a detectable $\mathrm{H} \alpha$ emission, which is obviously not the case. In general, it appears that the largest discrepancies between the "H $\alpha$ " and "UV" rates occur for galaxies with low SFRs, especially when B04 estimate SFRs based on calibrations that employed SF galaxies.

B04 SFRs are the sum of SFRs within and outside of the fiber. To trace the source of the SFR discrepancies, we revisit the calibrations used to derive B04 SFRs for non-SF classes. First, within the fiber, B04 base the SFRs for non-SF classes on the relation be-

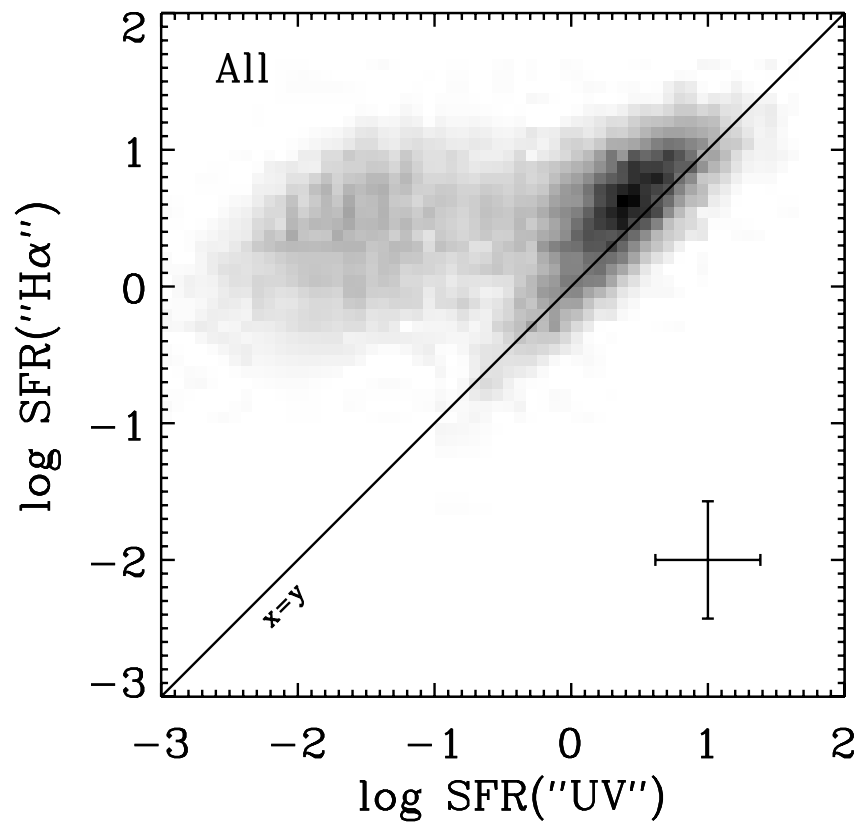

FIG. 2.-Comparison of dust-corrected SFRs derived by Brinchmann et al. (2004) (B04, "H $\alpha$ ") and the dust-corrected SFRs from this study ("UV"). Comparison is given for all galaxies in the sample. Our ("UV") SFRs come from the modeling of the broadband SED (two GALEX UV bands and five SDSS optical bands), and span almost 5 orders of magnitude. While most galaxies compare reasonably well, those with low "UV" SFRs are quite offset from the equality line. The error bar represents average formal errors (from Bayesian fitting) of the two estimates. Both estimates are given for (Chabrier 2003) IMF.

tween the specific SFR (in the fiber) and the D4000 index calibrated using the SF and low-S/N SF galaxies. We show this calibration in both panels of Figure 4 as the dashed line (this can be compared to their Fig. 11). Then, B04 use D4000 to determine the specific SFR (and therefore the SFR itself) of galaxies of other classes. In the top panel of Figure 4, we compare B04 relationship to our ("UV") relationships for the star-forming (bold solid line) and the low-S/N star-forming (thin solid line) galaxies. Note that in principle we cannot compare the B04 specific SFR inside of fiber to the total specific SFRs obtained from the UV, so the comparison is more for illustrative purposes. At D4000 $<1.7$ either SF or low-S/N SF lines from "UV" compares well to B04. However, notice that there are no galaxies classified as star-forming beyond $\mathrm{D} 4000=1.9$. Therefore, from that point on, B04 relationship is based only on low-S/N SF class, which are most likely just AGN contaminants (see $\S 3.2$ ). In contrast to B04, the "UV" relationship for low $\mathrm{S} / \mathrm{N}$ SF continues to decline as D4000 increases. In the bottom panel of Figure 4 we show the same B04 calibration against "UV" rates for the AGNs (thin solid line) and galaxies with no $\mathrm{H} \alpha$ detections (thick solid line). In these cases, the relationship for these two types as indicated by the "UV" is entirely different from that of the star-forming galaxies, even at lower D4000 values. For clarity we omit showing the composite class. Altogether, this leads to a conclusion that the relationship between the specific SFR and the D4000 index is not unique for different classes of galaxies. We tested if this is an artifact of the fact that D4000 comes from the central $3^{\prime \prime}$, while the specific SFR for "UV" is integral. We find that the relationships are qualitatively the same for $z<0.07$ and for $z>0.12$ samples for which D4000 probes different physical sizes. Instead, the most likely explanation is that since D4000 and specific SFR are sensitive to SF activity on different timescales, the galaxies with differing SF histories will have different relationships. To much lesser extent, 


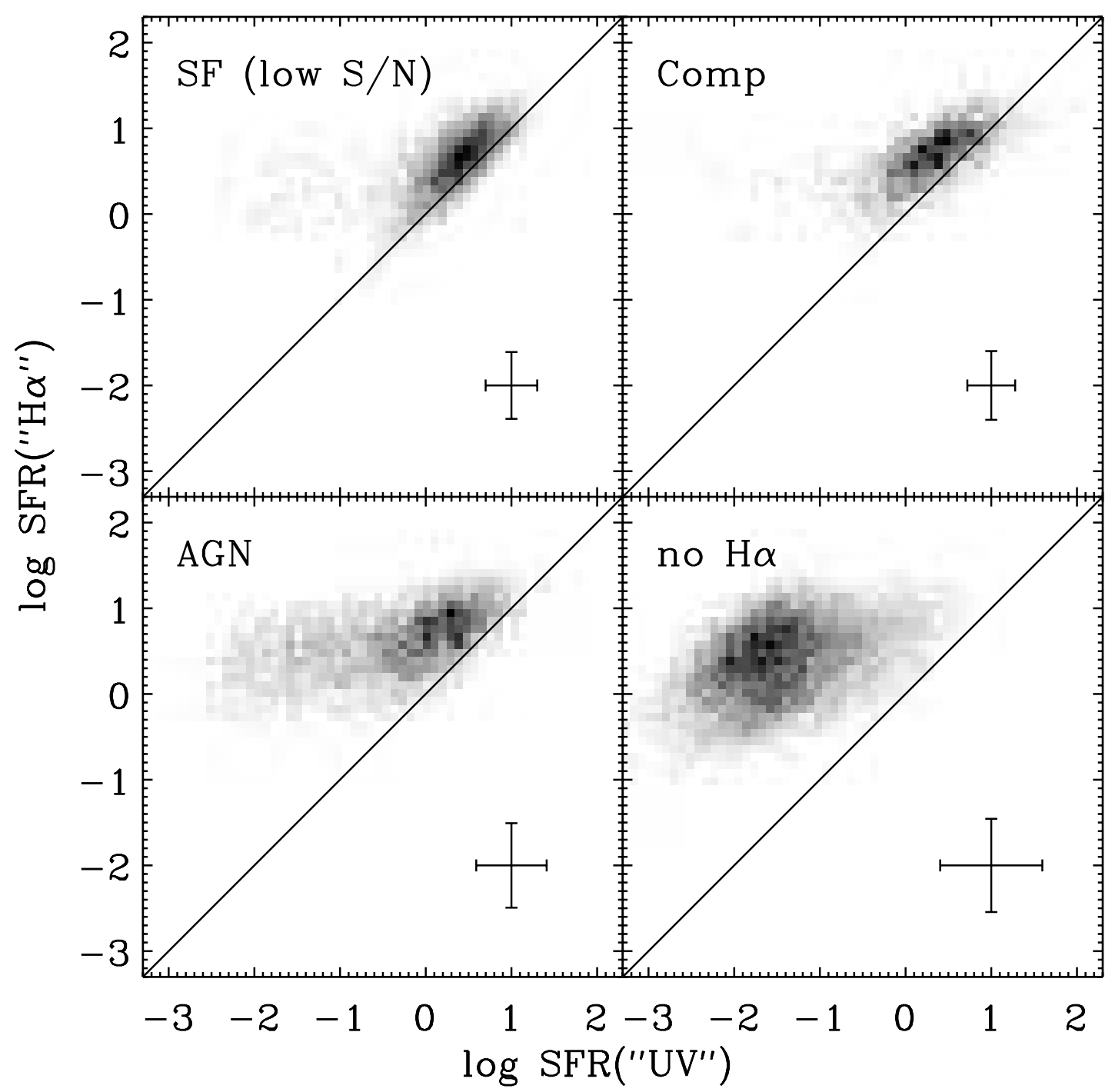

FIG. 3.-Comparison of B04 ("H $\alpha$ ") and "UV" dust-corrected SFRs for low-S/N star-forming, SF/AGN composite, AGNs, and galaxies without H $\alpha$ detection. Note that except for low-S/N SF class, the B04 estimate comes from relations calibrated using the "H $\alpha$ " SFRs of galaxies classified as star forming. In any class the discrepancies are particularly large when the "UV" estimate of SFR is low. Error bars represents average errors in each class.

some of the differences could be due to metallicity (Poggianti \& Barbaro 1997).

Given the level of aperture corrections (a factor of several), the analysis given above does not provide the explanation for the large discrepancies we see when comparing the total SFRs. Since the SFR outside of fiber dominates in B04 total SFR, we now focus on the calibration B04 use to determine it. As outlined in $\S 5.2 \mathrm{~B} 04$ aperture correction relies on the calibration of the luminosity-normalized SFR as a function of $g-r$ and $r-i$ color. Using our UV-based SFRs we can test one of the two assumptions behind B04 aperture corrections - whether the SFR $/ L$ calibration against color holds for different classes of galaxies. In Figure 5 we plot SFR/L against two colors that B04 use to determine SFRs outside offiber. The bold line represents the relation from galaxies classified as star forming. This is the basis for the B04 calibration that they apply to other classes as well. The dashed and the thin solid lines show the relations for AGNs and galaxies with no $\mathrm{H} \alpha$. We see (left panel) that as the $g-r$ color increases, the discrepancy between SF and other classes rises, and reaches some 0.8 dex for AGNs and 1.3 dex for $\mathrm{No} \mathrm{H} \alpha$. Similar levels of difference are present against $r-i$ (right panel). The level of these differences matches the discrepancies (plumes) in the comparison of B04 and
"UV" SFRs in Figure 3. We can interpret these differences as reflecting the fact that the colors used for B04 calibration trace old populations, while the current SFR traces young populations, and it is not surprising that the two will differ for different classes of galaxies. Also note that the discrepancies of specific SFRs of different classes of galaxies are qualitatively similar in the case of D4000 and in the case of $g-r$ color, which is not surprising since they are both sensitive to population age on the similar timescales, and are similarly not affected by the dust. To conclude, UV-derived SFR is subject to fewer limitations, so it can be applied to a more diverse types of normal galaxies.

\subsection{Comparison of "UV" and " $\mathrm{H} \alpha$ " SFRs for Star-forming Galaxies}

To avoid the problems indicated in the previous section, we need to compare the SFR estimates from the "H $\alpha$ " and the "UV" methods in galaxies where $\mathrm{H} \alpha$ is well-detected $(\mathrm{S} / \mathrm{N}>3)$ and arises predominantly from star formation, i.e., to galaxies classified as star forming (SF). In Figure 6 we show the B04 "H $\alpha$ " star formation rates plotted against our "UV." We see that the comparison, spanning some 3 orders of magnitude in SFR, is exceptionally good. Formal error bars are comparable, with the "UV" 


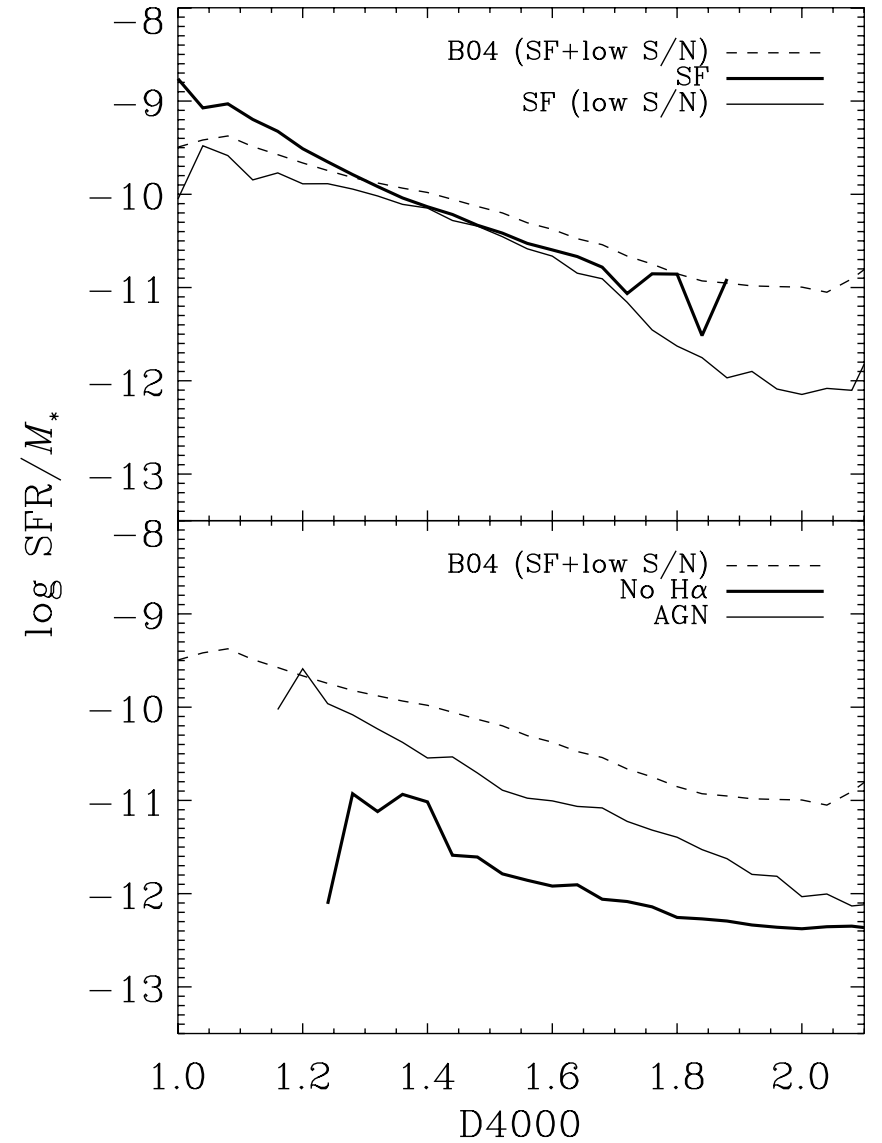

FIG. 4.-Relationships between SFR/ $M_{*}$ (the specific SFR) and the D4000 spectral index. In the top panel we compare the relationship from B04 (constructed from SF and low-S/N SF galaxies combined) to our "UV" relationships for the SF and the low-S/N SF class, separately. The B04 relationship comes from measurements within the fiber, while others are total, so this comparison is primary an illustration. B04 use such relationship to determine fiber SFRs of non-SF classes of galaxies. Note that beyond D4000 $=1.9$, the B04 calibration is actually based on low $\mathrm{S} / \mathrm{N}$ spectra, which may be contaminated by AGNs. In the bottom panel the same B04 relationship is compared to our "UV" rates for the AGNs, and for galaxies with no $\mathrm{H} \alpha$ detection. The two follow different relationships, with AGNs and "No $\mathrm{H} \alpha$ " classes having much lower specific SFRs than the SF class. The nonunique mapping between the specific SFR and the D4000 index for various galaxy groups, and the fact that the correlation cannot be established well using the emission lines indicates that fiber SFRs in B04 may be systematically affected.

being somewhat smaller (see Table 1). The scatter (standard deviation of the difference) of the two measurements is $0.50 \mathrm{dex}$. When $3 \sigma$ outliers are excluded, the scatter is reduced to $0.36 \mathrm{dex}$. This is very well matched by the sum (in quadrature) of the formal errors of the two methods ( $0.35 \mathrm{dex})$, confirming that the two measures are predominantly independent. There is an average offset between the two SFRs of only 0.06 dex in the sense that " $\mathrm{H} \alpha$ " SFR is higher (which reduces to 0.02 dex, i.e., $5 \%$, when $3 \sigma$ outliers are excluded).

However, Figure 6 alone can potentially hide some systematic trends between the two SFR estimates. Where can the differences in the SFR estimates arise from? Deriving SFRs for either methods incorporates several steps: (1) obtaining full "H $\alpha$ " or "UV" observed luminosity (which in the case of " $\mathrm{H} \alpha$ " involves aperture corrections), (2) correcting the observed luminosity for dust attenuation, and (3) converting the luminosity into a SFR. While both methods perform these three steps simultaneously, we can still design tests that would differentiate between. Given in some cases the large aperture correction applied by B04, we first plot in Figure 7 the difference in SFR estimates (SFR residuals) with respect

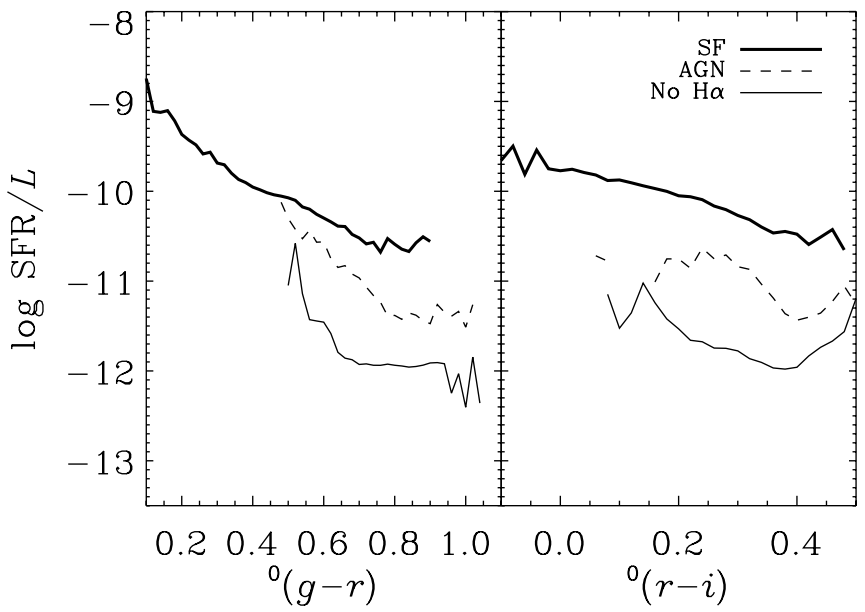

FIG. 5.- Relationship between the luminosity-normalized SFR and optical colors. B04 use luminosity-normalized SFRs binned in $g-r$ vs. $r-i$ color-color grid to estimate SFR outside of fiber. They build their relationships using SF galaxies, but apply them to other types as well. Here, based on "UV" measurements, we show that different classes of galaxies follow different relationships, and that applying the one from SF galaxies would lead to systematic differences, which cause most of the SFR discrepancies seen in Fig. 3.

to the level of aperture correction applied to " $\mathrm{H} \alpha$ " rates. We see no correlation. We also check, but do not find a correlation between the residuals and the apparent sizes of galaxies (not shown). Those are good indications that the aperture corrections of B04 are quite reliable for galaxies classified as star forming. Finally, we find that the SFR residuals exist when plotted against the stellar mass of a galaxy (shown in Figure 8). We have a change of SFR residual of 0.38 dex over the $8.5<\log M_{*}<11$ range, with $1 \sigma$ dispersion of $0.33-0.41$ dex around the running mean. To double check if the residuals are in any way connected with B04 aperture corrections, we limit the sample to farther, and therefore on average smaller galaxies $(z>0.12)$. We still find that the residuals correlate with stellar mass.

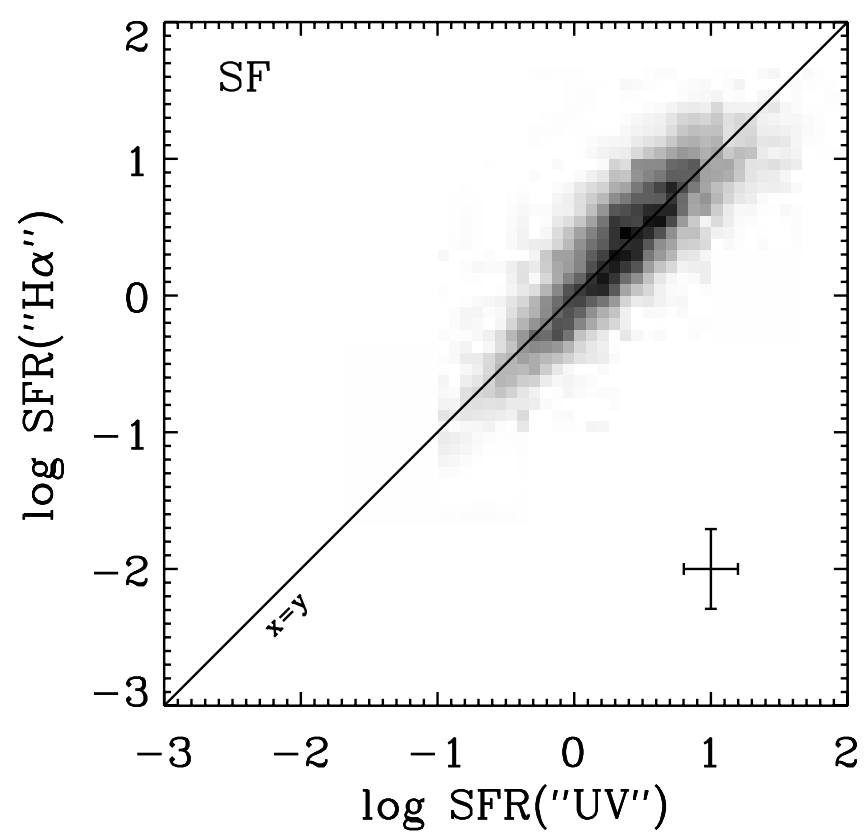

FIG. 6.-Comparison of B04 ("H $\alpha$ ") to our "UV" SFRs (both independently dust-corrected) for galaxies classified as star forming. The two compare very well on one-to-one basis. Also, the scatter is compatible with each measurement's errors. 


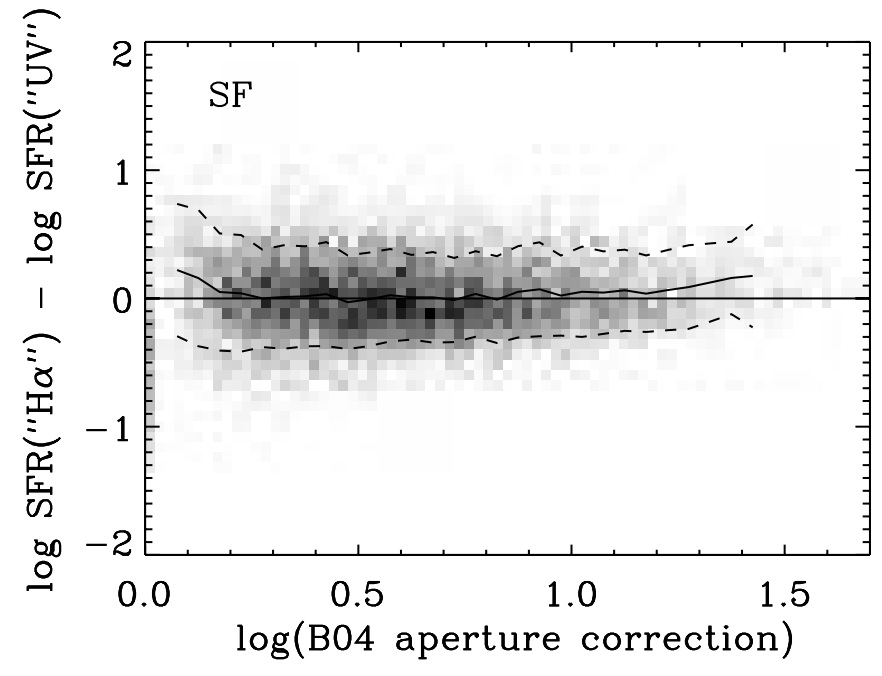

FIG. 7.-Difference of B04 ("H $\alpha$ ") and our "UV" SFRs, for galaxies classified as star forming, as a function of aperture correction applied to B04 SFRs. There is no apparent trend, suggesting that the aperture corrections derived by B04 are quite robust. Running average (in 0.05 dex bins) is shown as a thick line, and the $\pm 1 \sigma$ range as dashed lines.

Before ruling out step 1 as being responsible for the massdependent residuals, we perform two additional checks. First, imagine there is an offset in zero points between GALEX and SDSS magnitudes (after all, they come from different surveys and are not measured in identical apertures). For example, if GALEX fluxes were systematically overestimated, this could cause "UV" SFRs to be overestimated as well, possibly in such a way to preferentially boost blue, low-mass galaxies (as the trend in Fig. 8 would suggest). To test this, we perform full SED-fitting runs in which we make FUV and NUV fainter by $0.1,0.2$, and 0.4 mag. We first check the quality of these new fits by comparing the distribution of $\chi_{\text {best }}^{2}$ values with the nominal (no magnitude offset) run. Runs with 0.2 and 0.4 mag offsets produce evidently inferior fits, already suggesting that any potential offset cannot be that large. The run with 0.1 mag offset, however, appears as good as the nominal run, with an even slightly smaller average $\chi_{\text {best }}^{2}$. However, comparing the "UV" SFRs produced with offset GALEX magnitudes to "H $\alpha$ " SFRs, we find that while slightly flattening the slope of the residuals with respect to mass, the trend is not eliminated. In fact, the scatter of the residuals around the mean is larger when modified "UV" SFRs are used instead of nominal. We also look at the quality of fits with GALEX magnitudes offset in the opposite direction (making them brighter), but such fits are evidently inferior.

The second test concerns SDSS magnitudes. Namely, B04 use CMODEL SDSS magnitudes to transform fiber SFRs into total SFRs, while we use MODEL SDSS magnitudes when performing SED fitting. ${ }^{19}$ Therefore, we perform another SED fitting using CMODEL magnitudes instead. First, we notice that the quality of fitting with CMODEL magnitudes is noticeably inferior, stressing their inadequacy for producing reliable color estimates. Also, the use of CMODEL magnitudes does not remove the trend of SFR residuals, and the scatter of the residuals becomes larger than in the nominal

19 SDSS MODEL magnitudes (defined as either the exponential or de Vaucouleurs magnitude, depending on which profile better describes a galaxy) are preferred magnitudes for SED fitting as they preserve relative fluxes (colors) better. CMODEL magnitudes (defined as a composite of exponential and de Vaucouleurs magnitude) should provide a good measure of a total galaxy light.

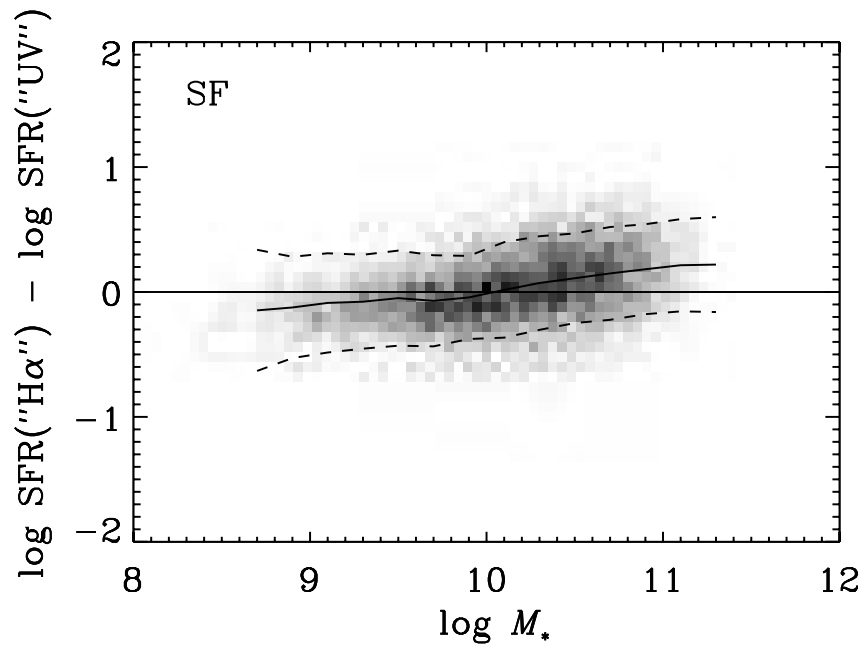

FIG. 8.-Difference of B04 ("H $\alpha$ ") and our "UV" SFRs, for galaxies classified as star forming, as a function of galaxy stellar mass. There is a clear trend with respect to mass, leading to a difference of SFRs of $0.38 \mathrm{dex}$ (a factor of 2.4) over the $8.5<\log M_{*}<11$ range. Running average (in 0.2 dex bins) is shown as a thick line, and the $\pm 1 \sigma$ range as dashed lines.

run, especially at higher masses. With this test we exhaust the possibilities that discrepancies arise in step 1 above.

We now move onto step 2, i.e., correcting the observed flux for dust attenuation. Note that both B04 and this study use Charlot \& Fall (2000) prescription for dust attenuation, the aim of which was to produce consistent treatment for $\mathrm{H} \alpha$, and UV continuum attenuation. Is the presence of the SFR residuals an indication that this dust attenuation model does not produce fully consistent answers? In our SED fitting we keep track of estimates on $\tau_{V}$ - the dust opacity in rest-frame $V$ band. We can thus compare our $\tau_{V}$ values with those obtained by B04 (which we denote $\tau_{V}$ ["UV"] and $\tau_{V}$ ["H $\alpha$ "], respectively). While we use the same model to constrain attenuations, B04 obtain them from the emission lines (to first order from the Balmer decrement), while we obtain them from the broadband SED (to first order from the UV spectral slope, $\beta$, or equivalently, the UV color). Also, B04 measurements are restricted to the fraction of the galaxy inside of the fiber, which can in principle produce some systematic differences. Direct one-toone comparison, shown in Figure 9, shows a rough agreement, albeit with a large scatter due to relatively large errors of both estimates (average formal errors shown as the error bar). Looking instead at the distributions of the two $\tau_{V}$ estimates (not shown), we find them to be very similar on the whole, with the $\tau_{V}$ ("UV") slightly offset toward the larger values with respect to $\tau_{V}$ (" $\mathrm{H} \alpha$ "). Since we find SFR residuals to correlate with the mass, it is more instructive to check whether such a trend exists in the difference of $\tau_{V}$ estimates from "H $\alpha$ " and "UV." In Figure 10 we plot $\tau_{V}$ residuals against the stellar mass. Despite the large scatter, we find a systematic trend. The gradient is 0.47 (equivalent to $0.52 \mathrm{mag}$ ) over the $8.5<\log M_{*}<11$ range. For lower masses, "UV" $\tau_{V}$ attenuation is higher than " $\mathrm{H} \alpha$," which implies that the observed UV flux is corrected more than $\tau_{V}$ ("H $\alpha$ ") would require, resulting in "UV" SFRs being higher than SFR("H $\alpha$ "). The situation is reversed at high masses. This trend matches the sense of the trend of SFR residuals.

In order to establish whether the difference in $\tau_{V}$ estimates is the dominant cause of the trend of SFR residuals, we try to modify one of the two SFR estimates (e.g., the "UV" SFR) based on the difference in $\tau_{V}$ values. Note that this is an approximate technique, since our SED SFRs are not "corrected" for dust by the 


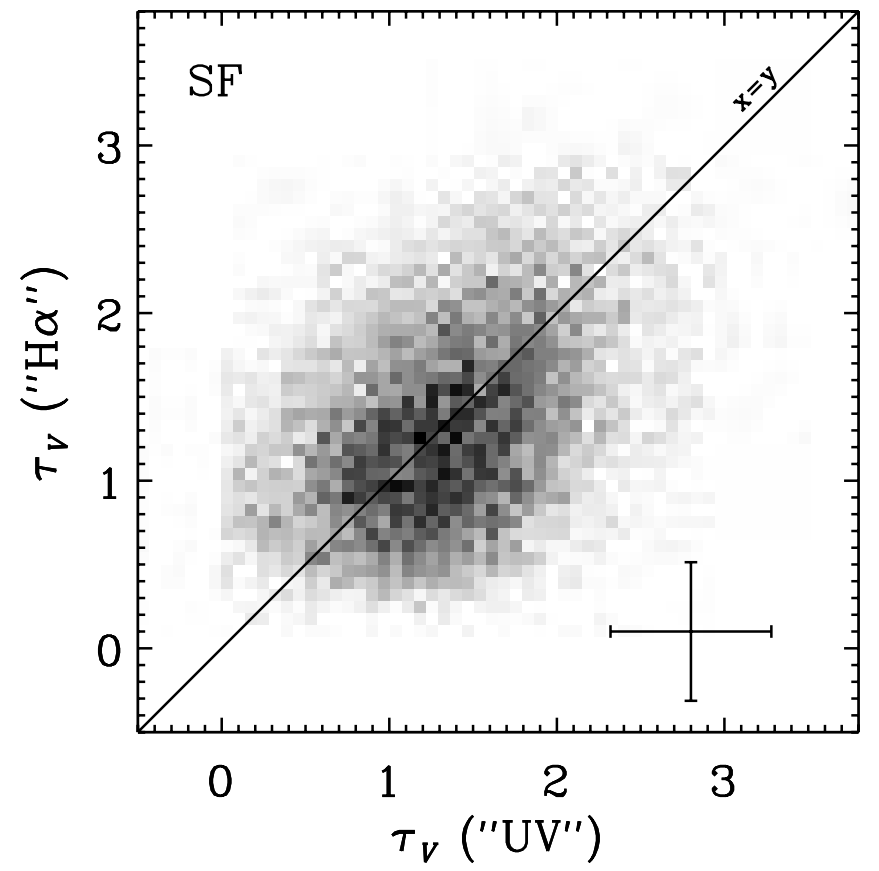

FIG. 9.-Comparison of B04 ("H $\alpha$ ") to our ("UV") estimates of $V$-band dust opacity (attenuation), for the SF galaxy class. The formal error of both estimates (average errors shown) is large, leading to a large scatter. However, there is an agreement between the two in the general sense. B04 $\tau_{V}$ is to first order constrained by the $\mathrm{H} \alpha / \mathrm{H} \beta$ ratio (Balmer decrement), while our estimate is predominately constrained by the UV slope. Both estimates were made in accordance with the Charlot \& Fall (2000) two-component dust attenuation model.

application of a single number, but rather by a complex application of the Charlot \& Fall (2000) prescription on many different populations that constitute the model SED. Nevertheless, for the purposes of this test we would assume that the attenuation in the FUV drives the correction of the "UV" SFR estimate. We therefore modify it with

$$
\mathrm{SFR}(\text { ('UV') })_{\bmod }=\mathrm{SFR}(\text { ('UV') }) \times 10^{0.4 \Delta A_{\mathrm{FUV}}}
$$

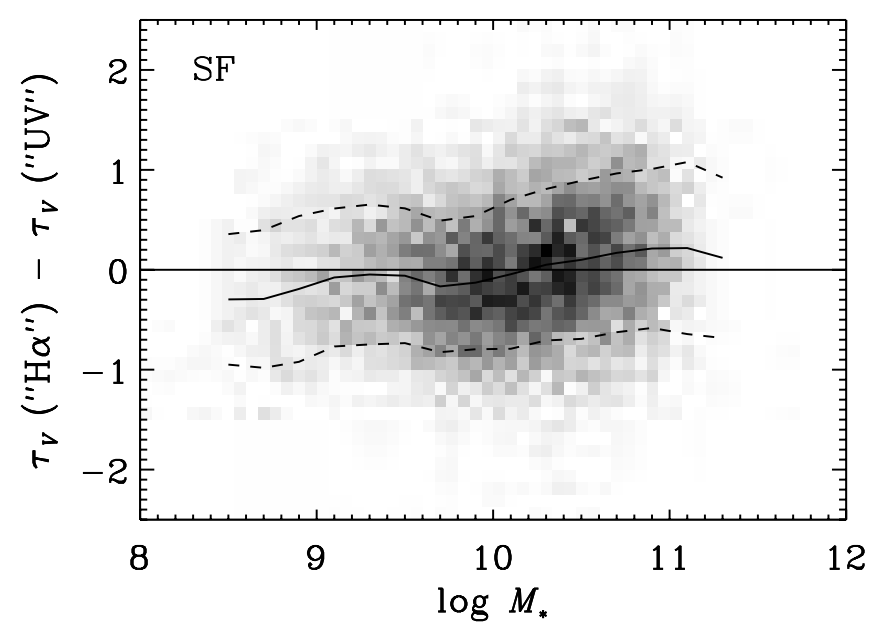

FIG. 10.-Difference of B04 ("H $\alpha$ ") and our "UV" estimates of $V$-band dust opacity as a function of galaxy stellar mass (for SF galaxies). There is a trend with respect to mass, leading to a 0.5 difference over the $8.5<\log M_{*}<11$ range. Running average (in 0.2 dex bins) is shown as a thick line, and the $\pm 1 \sigma$ range as dashed lines.

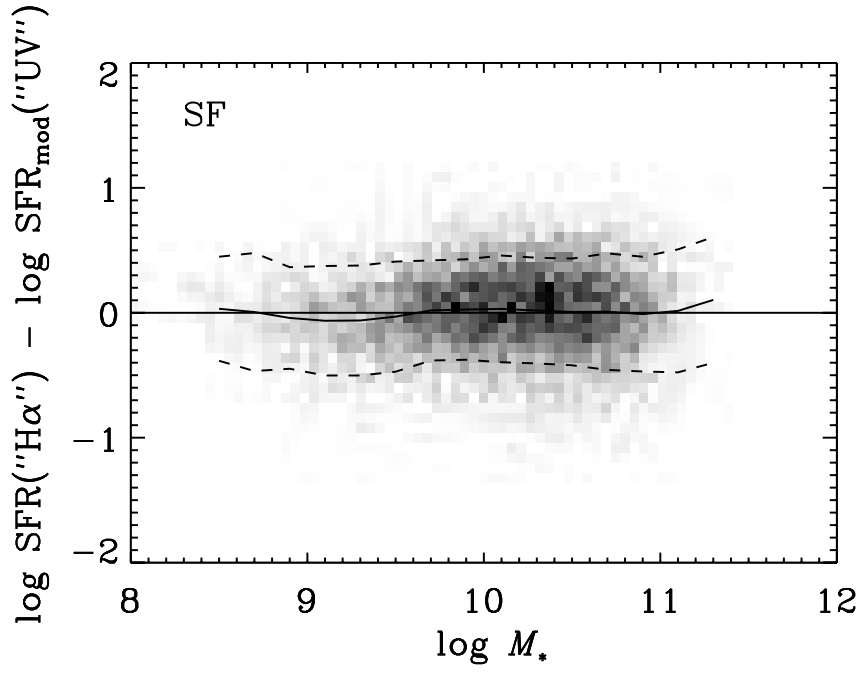

FIG. 11.-Difference of B04 ("H $\alpha$ ") and the modified "UV" SFRs as a function of galaxy stellar mass. We modify one of the two SFRs (in this case the "UV" SFR) by the difference in B04 and our attenuation estimate. After this modification, the trend of the residuals with respect to mass is eliminated, and the overall difference is close to zero. Therefore, we find that emission lines and the continuum essentially produce the same SFR estimates. Running average (in 0.2 dex bins) is shown as a thick line, and the $\pm 1 \sigma$ range as dashed lines.

where $\Delta A_{\mathrm{FUV}}$ is the difference between the attenuation in the rest-frame FUV as implied by $\tau_{V}$ ("H $\alpha$ ") and that determined from the SED fitting. We look at the parameters from the SED fitting to calibrate the relationship between the attenuation in FUV and the $V$-band opacity, and find the following relationship:

$$
\Delta A_{\mathrm{FUV}}=\mu\left(5.4-0.84 \tau_{V}\right) \Delta \tau_{V}
$$

where $\Delta \tau_{V}=\tau_{V}$ ("'H $\alpha$ ") $)-\tau_{V}$ (" $\mathrm{UV}$ '), while $\tau_{V}$ and $\mu$ come from the SED fitting (i.e., they are "UV" measurements). The parameter $\mu$ is the coefficient in the Charlot \& Fall (2000) model that determines the fraction of the total opacity affecting the diffuse ISM. We modify SFR("UV") according to equations (3) and (4), and in Figure 11 replot the difference in SFRs against the mass. The trend of the SFR residuals with mass has disappeared, and the difference stays within $\pm 10 \%$ across the full mass range. The dispersion around the running mean is $20 \%$ larger than with the nonmodified SFR residuals, which is to be expected due to the approximate nature of the modification applied. Therefore, we conclude that the "UV" continuum and the "H $\alpha$ " in essence produce identical answers for the star formation rate, the apparent difference stemming from different estimates of the attenuation that the two methods provide. With this in mind, we can also rule out other possibilities for the cause of the original differences in the SFRs, such as the reliability of converting the luminosity into a SFR (point 3 above). Also, these results show that, contrary to some notions, the Bruzual \& Charlot (2003) models cannot be too far off in the UV.

Although we can now account for the SFR differences, we would still like to understand the origin of attenuation differences that cause them. Related to this, we should try to determine which attenuation estimate (emission-line or the UV continuum) is more accurate. At this point we need to emphasize that our SED fitting has a nonflat prior distribution of $\tau_{V}$ (described in $\S 4.1$ ), while B04 uses a flat prior, with attenuation taking values in the $0.01<$ $\tau_{V}<4$ range. While the nonflat prior used in our study is a reasonable assumption, and the one that helps constrain the parameter 


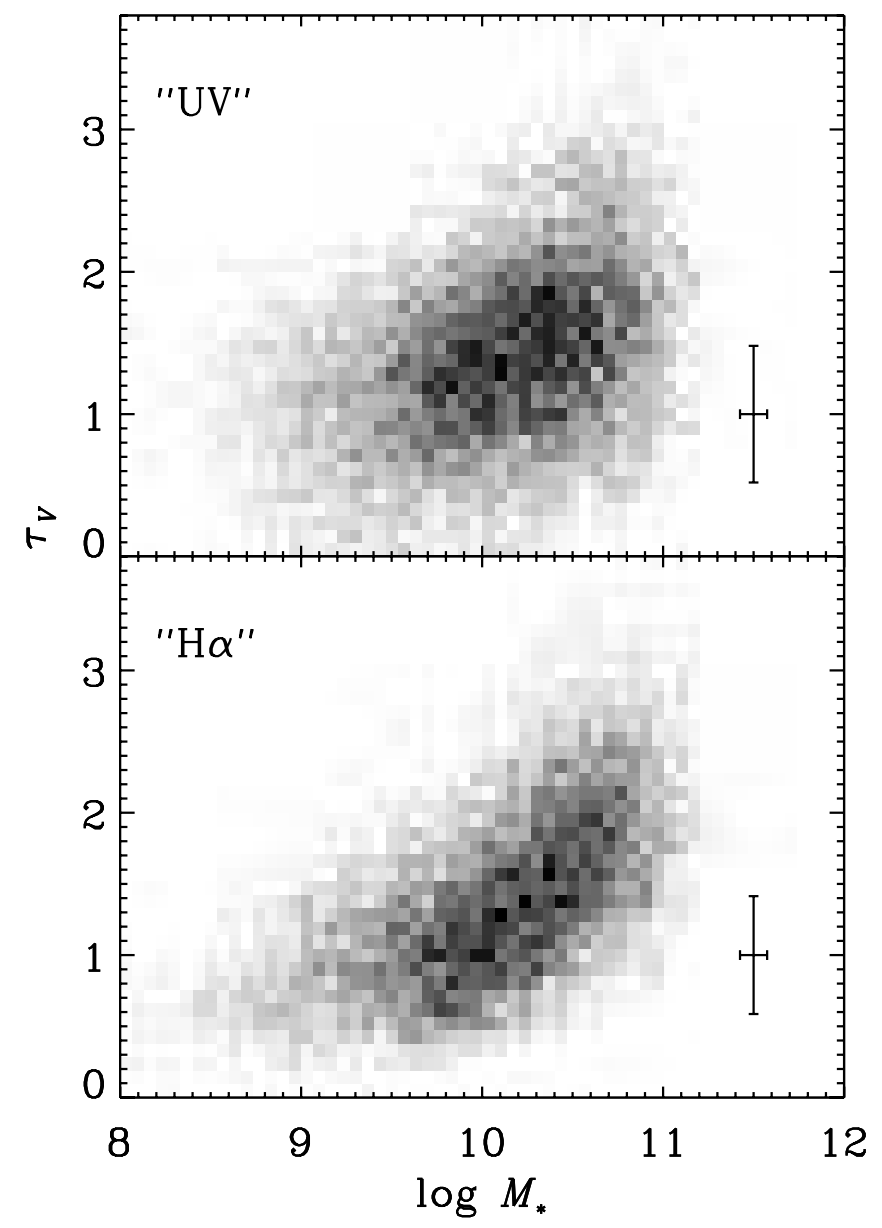

FIG. 12.-Attenuation-mass relationship for star-forming galaxies. The two attenuation estimates, one from our SED fitting ("UV"; top), and the other from B04 ("H $\alpha$ "; bottom) are plotted against the stellar mass. The B04 estimate (principally from $\mathrm{H} \alpha / \mathrm{H} \beta$ ratio) produces a tighter relationship with a greater span of attenuation values, indicating that it is probably more accurate.

space to physically realistic values, we would like to check whether the differences in priors induce any systematic effects. To that effect we first perform an additional SED fitting run, with the model libraries created with a flat attenuation prior, taking values in the $0<\tau_{V}<3$ range. At lower $\left(\tau_{V}<0.7\right)$ values, we find the distribution of the resulting $\tau_{V}$ values to be similar to the original distribution, while at higher values it displaces the original peak, located at $\tau_{V} \approx 1.3$, to a broader distribution peaking at $\tau_{V} \approx 2.0$. The latter is the obvious consequence of assigning equal probability to models with high attenuations. In any case, we end up with a $\tau_{V}$ distribution that differs more with respect to the B04 $\tau_{V}$ ("H $\alpha$ ") than did the original $\tau_{V}$ ("UV"). Nevertheless, we proceed and check the relationship between the new SFR residuals and the stellar mass. The trend observed in the original relationship is still present, although it is somewhat weaker at low masses. Anyhow, it appears that we cannot force the two attenuation estimates to reach an exact agreement by a simple modification of the prior distribution used in SED fitting.

Next we try to get a sense of which $\tau_{V}$ estimate is more realistic. Since the direct comparison with an external independent measurement is not readily available, we will try to evaluate the differences using internal relations. For star-forming galaxies we expect attenuation to be correlated with the stellar mass. In Figure 12 we show this relation for our "UV" estimates (top panel) and $\mathrm{B} 04$ "H $\alpha$ " estimates (bottom panel). It is apparent that the latter defines a more tight relationship. Also, $\tau_{V}$ ("H $\alpha$ ") spans a larger range of values than $\tau_{V}$ ("UV") (1.4 vs. 0.8 over $8.5<$ $\left.\log M_{*}<11\right)$. Finally, the dispersion around a running mean is some $20 \%$ smaller for $\tau_{V}$ ("H $\alpha$ "). This all indicates that B04 $\tau_{V}$ estimates are probably more accurate. While the two $\tau_{V}$ estimates agree well on the whole (Fig. 9), the "UV" estimate appears more poorly constrained, which may cause it to more often take values away from the extrema, suppressing the span in relationship with the mass. As a result, the attenuation estimates for low-mass galaxies will be overestimated, and for high-mass galaxies underestimated. It is difficult to directly test this explanation. Namely, if we restrict the analysis only to galaxies with small formal errors in $\tau_{V}$ ("UV") (or alternatively small errors in FUV - NUV), we at the same time bias the sample to smaller values of $\tau_{V}$ ("UV"). Alternative explanation is that the Charlot \& Fall $(2000) \propto \lambda^{-0.7}$ extinction curve deviates from the true extinction curve in a way that would be mass-dependent (or some quantity related to mass, such as the metallicity). A possibility that the extinction curve is mass-dependent is raised in Johnson et al. (2007), but no significant effect has been found. We would be able to acquire further insight by comparing both attenuation estimates to some external measure, such as the IR excess (IRX). Johnson et al. (2007) have recently obtained IRX attenuations from Spitzer MIPS and GALEX observations of SDSS galaxies, therefore such a sample can be used to include UV (i.e., SED) derived attenuations. Also, the SEDbased attenuation estimates can possibly be improved by adding the near-IR data, such as the $J H K_{s}$ photometry from 2MASS survey (Skrutskie et al. 2006), which would improve attenuation estimates by placing stronger constraints on the stellar metallicity.

\section{OBTAINING THE UV SFRS WITHOUT SED MODELING}

In this work we are using sophisticated SED modeling to obtain UV-based star formation rates. In many applications such a detailed approach is not practical, or not even possible. In such cases one would like to obtain a reasonably good estimate of a dust-corrected star formation by applying some simple transformations to the UV photometry. While such methods have been used in many previous studies, here we will use the results of the detailed SED analysis to calibrate such simplified models, with a special emphasis on users of UV data obtained with GALEX. Since for the foreseeable future GALEX will remain the only facility capable of observing a large number of galaxies in the UV, it is not without justification to treat its filters as defining some standard photometric bands in the UV domain. Researchers who study high-z galaxies in rest-frame UV can calibrate their blueshifted filters against GALEX FUV and NUV response curves. ${ }^{20}$

Obtaining a UV-based SFR consists of $K$-correcting the UV magnitudes, estimating the dust attenuation of the FUV flux, and converting the dust-corrected FUV luminosity into a SFR. We assume that the user will correct their data for the Galactic extinction, and then apply some standard $K$-correction procedure to obtain rest-frame FUV and NUV magnitudes. Of course, obtaining reliable $K$-corrections requires optical or near-IR photometry. To calibrate the relations in this section, we use KCORRECT v4_1_4 (§ 4.4).

We start by using the results of the full SED fitting to calibrate the well-known correlation between the attenuation and the UV spectral slope (e.g., Calzetti et al. 1994). This is often refereed to as the IRX- $\beta$ relation, although strictly speaking IRX indicates IR excess, which is correlated with UV attenuation. IRX- $\beta$

\footnotetext{
${ }^{20}$ GALEX filter response curves can be obtained from http://galexgi.gsfc.nasa .gov/tools/Resolution_Response.
} 


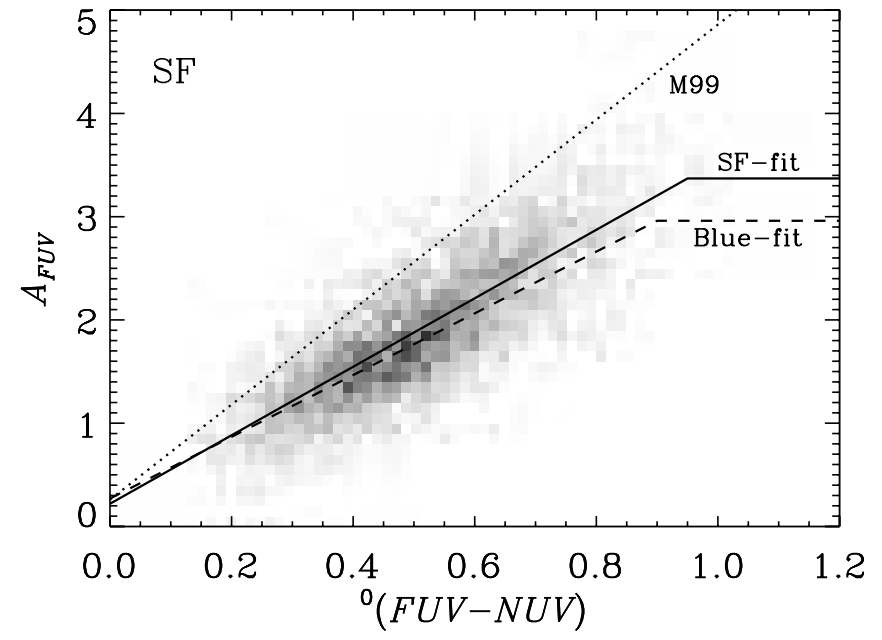

FIG. 13.-Relationship between the attenuation in the GALEX far-UV and the UV slope. The FUV attenuation estimate comes from our SED fitting, and the FUV - NUV rest-frame color serves as an indicator of the UV SED slope. Data and the solid line linear fit is for galaxies classified as star forming. The dashed line is a fit to blue (rest-frame NUV $-r<4$ ) galaxies. The fits reproduce the trends in general, however, there is a significant scatter. Meurer et al. (1999) relation for starburst galaxies is plotted as the dotted line (M99). It is obviously not appropriate for our sample of mostly normal star-forming galaxies.

relationship for normal galaxies, such as those in our sample, has been previously studied with GALEX data by Seibert et al. (2005), Cortese et al. (2006), Gil de Paz et al. (2007), Boissier et al. (2007), and Panuzzo et al. (2007). In Figure 13 we show FUV attenuations ( $\left.A_{\mathrm{FUV}}\right)$ obtained from our SED modeling, for galaxies classified as star forming, plotted against the rest-frame UV color, which is linearly correlated to the UV spectral slope. We see that the majority of objects lies along the ridge. This confirms that there exists an "IRX- $\beta$ " relationship for normal galaxies, not just for starburst galaxies as usually assumed. To quantify the relationship for our sample, and thus allow $A_{\mathrm{FUV}}$ to be determined from GALEX observations of normal galaxies, we fit a linear function to running medians (with value from each 0.05 mag bin weighted equally). Using the medians is necessary in order to avoid the fit to be affected by numerous outliers. Also, we find that past some red UV color the attenuation does not seem to rise, so we adopt a constant value. In any case, $A_{\mathrm{FUV}}$ exhibits a large scatter for such red colors.

$$
\begin{aligned}
& A_{\mathrm{FUV}}= \\
& \begin{cases}3.32^{0}(\mathrm{FUV}-\mathrm{NUV})+0.22, & { }^{0}(\mathrm{FUV}-\mathrm{NUV})<0.95, \\
3.37, & { }^{0}(\mathrm{FUV}-\mathrm{NUV}) \geq 0.95,\end{cases}
\end{aligned}
$$

where $A_{\mathrm{FUV}}$ is in magnitudes, and superscript 0 designates restframe colors. This relation is plotted in Figure 13 as a solid line. In many applications, especially at higher redshift, spectroscopic classification may not be available. In those cases one can select star-forming galaxies based on their color. For blue-sequence galaxies $\left({ }^{0}(\mathrm{NUV}-r)<4\right.$, and without applying any class selection) one should use a slightly modified relation:

$$
\begin{aligned}
& A_{\mathrm{FUV}}= \\
& \left\{\begin{array}{ll}
2.99^{0}(\mathrm{FUV}-\mathrm{NUV})+0.27, & { }^{0}(\mathrm{FUV}-\mathrm{NUV})<0.90 \\
2.96, & 0
\end{array},\right.
\end{aligned}
$$

shown in Figure 13 as a dashed line. Note that these relationships are optimized for GALEX bandpasses and for normal starforming galaxies. We overplot Meurer et al. (1999) relation as a dotted line (we used the Seibert et al. [2005] transformation to obtain FUV - NUV from the UV spectral slope). Apparently, this relation, constructed from a sample of starburst galaxies, is well above the majority of galaxies in our sample. Seibert et al. (2005) and Cortese et al. (2006) using smaller samples (RC3 and cluster galaxies, respectively) also find that Meurer et al. (1999) relation overpredicts FUV attenuation. Seibert et al. (2005) derive a relation that exactly bisects our SF fit and the Meurer et al. (1999) relation. Also note that these relations pertain to the optically selected sample (since practically all SF galaxies are detected in UV bands, there is no additional UV selection). In general, samples selected at different wavelengths will have different IRX- $\beta$ relations, as demonstrated by Buat et al. (2005) using GALEX NUV and IRAS $60 \mu \mathrm{m}$ selected samples.

For a source with known redshift, the dust-corrected, rest-frame FUV is converted into a FUV luminosity. The final step requires converting the luminosity into SFR. Following B04 notation, we define:

$$
\eta_{\mathrm{FUV}}^{0}=L_{\mathrm{FUV}}^{0} / \mathrm{SFR} \text { (“'UV'”), }
$$

to be the inverse conversion factor between a dust-corrected restframe FUV luminosity (in ergs ${ }^{-1} \mathrm{~Hz}^{-1}$ ) and the SED-derived SFR. The conversion factors comes from the stellar population modeling that was used to perform the SED fitting. Like the equivalent conversion factor for $\mathrm{H} \alpha$ luminosity, $\eta_{\mathrm{FUV}}^{0}$ is sensitive to metallicity, albeit more weakly so. For our sample (the metallicity of which is on average $0.8 Z_{\odot}$ ), the median conversion factor is

$$
\log \eta_{\mathrm{FUV}}^{0}=28.165
$$

The above factor is given for the Chabrier (2003) IMF, which is used in this paper. The often-used conversion factor given by Kennicutt (1998) for the Salpeter (1955) IMF is

$$
\mathrm{SFR}=1.4 \times 10^{-28} L_{\nu}
$$

where SFR is in $M_{\odot} \mathrm{yr}^{-1}$ and $L_{\nu}$ is in $\operatorname{ergs~s}^{-1} \mathrm{~Hz}^{-1}$.

We find using Bruzual \& Charlot (2003) models that the appropriate transformation factor between UV-derived SFRs that assume Chabrier and Salpeter IMFs is 1.58 (both with $0.1-100 M_{\odot}$ limits). ${ }^{21}$ Thus our "empirical" conversion factor (eq. [8]) for Salpeter IMF becomes

$$
\mathrm{SFR}=1.08 \times 10^{-28} L_{\mathrm{FUV}}^{0}
$$

where SFR and $L$ have the same units as in equation (9).

This implies that the Kennicutt (1998) conversion factor (eq. [9]) is $30 \%$ higher. We verify that the effect of the difference in bandpasses (the Kennicutt 1998 factor was given for the 1500$2800 \AA$ range, while the GALEX FUV filter spans 1300-1800 $\AA$ ) is completely negligible. The actual reasons for the difference are two-fold. First, even when obtaining the model conversion factor using Bruzual \& Charlot (2003) and the same assumptions as given by Kennicutt (1998; i.e., solar metallicity and a constant star formation history), we still find Kennicutt (1998) conversion to be $15 \%$ higher (i.e., the corresponding $\eta$ is lower than one produced by Bruzual \& Charlot 2003). Further, we have that our sample has

21 Note that the conversion between the IMFs is not generally the same for $\mathrm{H} \alpha$-derived SFR, UV SFR, or stellar mass. 


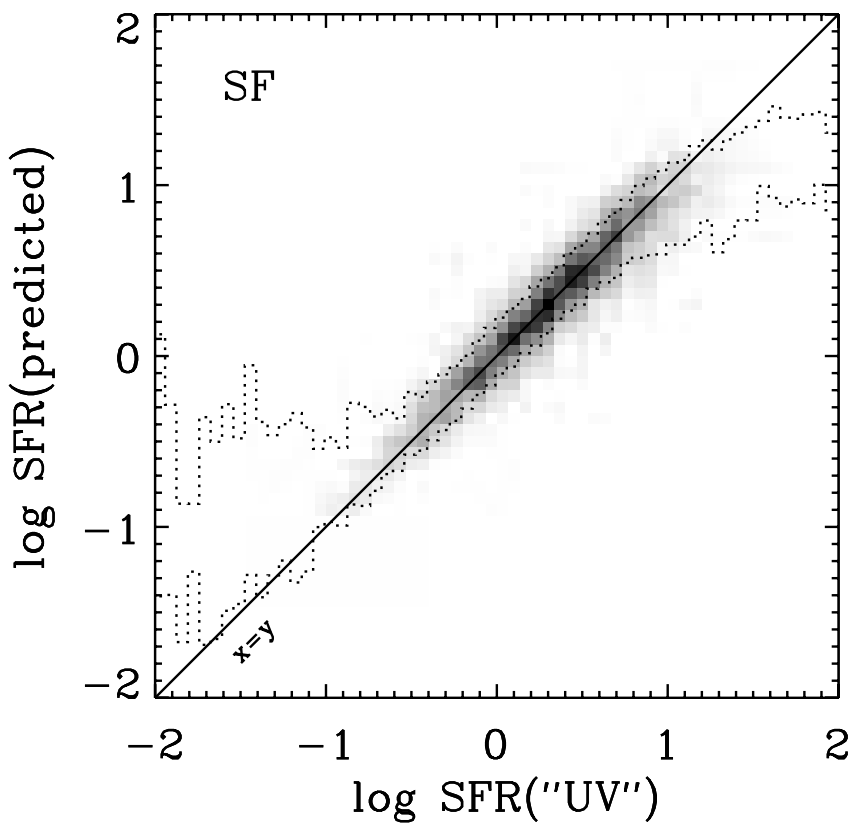

FIG. 14.-Comparison of the SFRs "predicted" using a set of simple transformations, to SFRs derived using the full SED fitting. The predicted SFR is obtained using the transformations that we calibrate from the SED SFRs. They are applied to K-corrected FUV and NUV magnitudes (see $\S 6$ ). The comparison is good for the majority of galaxies (grayscale scatter plot). The dashed lines show 16 th and 84 th percentile values of the conditional distribution at each SFR("UV"), from which we see that the predicted SFRs deviate from SFR("UV") at low and high SFRs.

average metallicity somewhat lower than the solar. This accounts for another 5\% difference. Finally, the remaining 10\% difference stems from the fact that our sample has a variety of star formation histories, giving $\eta$ that is on average higher than that for constant SF history. To summarize, for optically selected samples similar to ours, we suggest using "empirical" conversion given in equation (8) or (10).

Finally, we combine the above ingredients and "predict" the SFR from $K$-corrected FUV and NUV magnitudes. In Figure 14 we compare the SFR obtained using this simple prescription to the original SFR("UV"). We observe a good agreement for the majority of objects (the gray-scale scatter plot). In addition, for each bin in SFR("UV") we show a 16th-84th percentile range of the predicted SFR values, i.e., their conditional distribution. This allows us to track the trend in the regions where there are few points to show on the gray-scale scatter plot. We see that the predicted SFR starts to deviate at both the low and the high SFR ends, by up to 1 dex. Good agreement is obtained in the $-1<$ $\log$ SFR $<1$ range, i.e., where the majority of galaxies lie. The reason for the deviations at low and high-SFR end stem from the inability to estimate $A_{\mathrm{FUV}}$ reliably only from FUV and NUV for galaxies having extreme SF. No simple modifications of equations (5) and (6) are able to reproduce $A_{\mathrm{FUV}}$ estimate of galaxies with extreme SFRs without destroying the agreement for the majority of normal galaxies. In $-1<\log$ SFR $<1$ range, the 16 th -84 th percentile range is $0.35 \mathrm{dex}$ wide, indicating that the predicted values have an equivalent $1 \sigma$ scatter of 0.17 dex. Therefore, the total error of the predicted SFRs in this range is 0.26 dex (with the error of SFR ["UV"] itself added in quadrature). This is a small penalty considering the ease of the method. Note that the prescription given here is optimized to produce good results for the large majority of galaxies, and that a result for any given galaxy should be used with caution.

\section{STAR FORMATION IN THE LOCAL UNIVERSE}

\subsection{Star Formation History and Stellar Mass}

It is now commonly accepted that the star formation history of a given galaxy depends strongly on its mass (for early work based on UV see Boselli et al. 2001; Gavazzi \& Scodeggio 1996), and to some extent its environment. To first order, we can characterize the star formation history using the specific star formation rate, which is defined as the ratio of the current SFR to the current stellar mass. Thus, higher values of the specific SFR indicate that a larger fraction of stars was formed recently. The unit of specific SFR is inverse time, and it is thus often referred to as the galaxy build-up time. However, this should not be confused with an actual galaxy age, since the specific SFR tells us only how long it would have taken to build a galaxy assuming it had a current SFR throughout its lifetime, and assuming that the current stellar mass is close to the total mass formed in stars during a galaxy's lifetime (the two are not the same because of gas recycling).

For each galaxy in our sample we construct a two-dimensional probability distribution function in the $\left(\log \left(\mathrm{SFR} / M_{*}\right), \log M_{*}\right)$ plane as outlined in $\S 4$. We use a full two-dimensional PDF instead of collapsing it into a single value in order to better represent low-SFR galaxies whose PDFs are often very wide (i.e., not well constrained) in specific SFR, and in which cases the single value, such as the average of the marginalized PDF, would tend to artificially concentrate in the middle of the PDF. In Figure 15 we show the co-added two-dimensional PDFs in $0.1 / 0.05$ dex wide bins. In the top panel of Figure 15, as in all previous figures in the paper, we show unweighted values; i.e., each galaxy in our sample contributes equally to the gray-scale density. Given our redshift and magnitude limits, we adopt the $r$-band absolute magnitude limit of $M_{r}=-14.75$ (which we verify by constructing a luminosity function). This luminosity limit leads to the completeness in stellar mass of $\log M_{*} \approx 7.5$, which is below the plotted mass range. The dashed line is shown for reference and represents a constant SFR of $1 M_{\odot} \mathrm{yr}^{-1}$. We see that lower mass galaxies appear to be confined in a relatively narrow sequence that declines as the mass increases. At masses above $10^{10} M_{\odot}$, some galaxies assume a much lower value of the specific SFR; i.e., the sequence turns over. In the bottom panel, each galaxy's PDF is weighted by its $V_{\max }$ value; i.e., the sample is volume-corrected. Since the volume-corrected values span a very wide range of values, we now show logarithms of number densities, scaled so that the full gray-scale range displays densities from maximum (black) to 2 dex below the maximum value (lightest shade of gray). Each horizontal slice in effect represents a mass function of galaxies of a given specific SFR. It now becomes apparent that the sequence seen in the unweighted plot extends to lower masses, with an increasing number density of galaxies. Also, the mass distributions at low specific SFR extends to lower masses than implied by the noncorrected plot. Considering the size of the sample, the accuracy of SFRs, and the sensitivity to low levels of SF, this figure represents the best available assessment of the star formation history in the local universe. The typical error in stellar mass is only 0.07 dex, and 0.40 dex in the specific SFR (note that the errors in specific SFR vary by a factor of 3 across the sample). We complement the above figure with Figure 16 (top panel), where we display conditional probability distribution in each 0.05 dex wide mass bin, which is equivalent to normalizing each column to its maximum value. Such representation allows us to see what specific SFR dominates at a given mass, and it also displays more clearly the behavior at masses where there are too few objects to show on the standard plot. At masses below $10^{10} M_{\odot}$, high specific SFRs dominate. At $M_{*}=10^{10} M_{\odot}$, more galaxies assume 


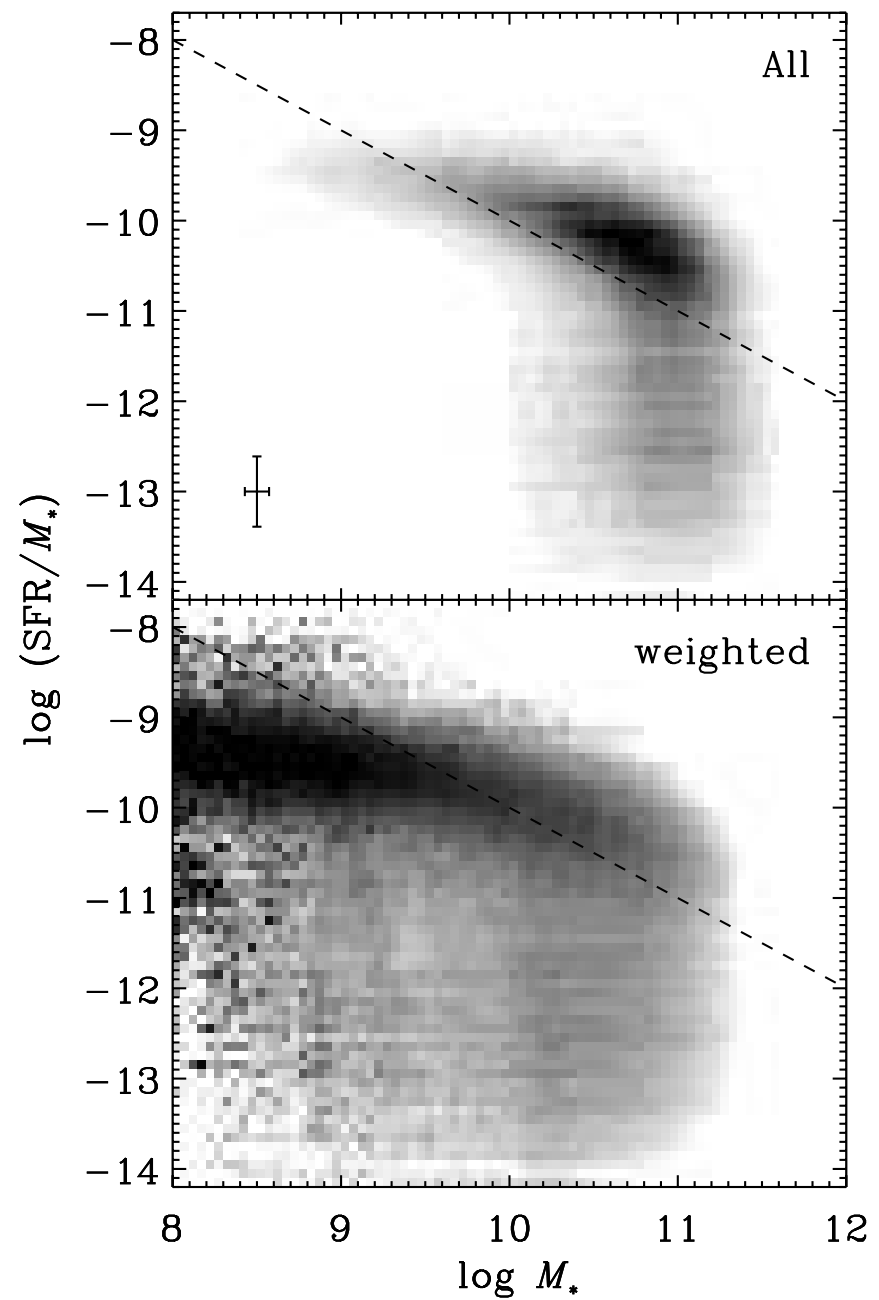

FIG. 15.-Dependence of the star formation history on the stellar mass. We use the specific SFR (SFR/ $M_{*}$ ) as an indicator of a star formation history. Galaxies with a larger fraction of recent star formation will have a higher value of the specific SFR. Instead of a single value, each galaxy is represented with a full two dimensional probability function. The dashed line, shown for reference, represents a constant SFR of $1 M_{\odot} \mathrm{yr}^{-1}$. The top panel gives equal weight to every galaxy in the sample, while the bottom panel shows logarithms of volume-corrected values (i.e., weighted by $V_{\max }$ ). Uneven behavior at low masses is because of a small number of galaxies (or no galaxies) in some bins. Note that the sample is only optically selected. Our completeness limit is below the lowest mass on the plot.

values below the dominant high-specific SFR "sequence," down to very low levels. At $M_{*}=2 \times 10^{11} M_{\odot}$, close to where the highspecific SFR "sequence" terminates, the galaxies with low specific SFRs begin to dominate. Above $\log M_{*}=11.8$ the plot is dominated by noise from very few objects with such high mass. This figure compares well to Figure 24 in B04.

\subsection{The Star-forming Sequence}

We now focus on star formation histories of various classes of galaxies. In Figure 17 we show specific SFR against the stellar mass for the star-forming galaxies, AGNs (together with SF/AGN composites), and the class without $\mathrm{H} \alpha$ detection. For each class the top panels show nominal, unweighted data, while the bottom panels are volume-corrected. Dashed line has the same meaning as in Figure 15. First we notice that the three classes occupy relatively distinct portions of the parameter space. This is especially pronounced in the unweighted plots. Thus, the three classes appear to have had quite different star formation histories. This was

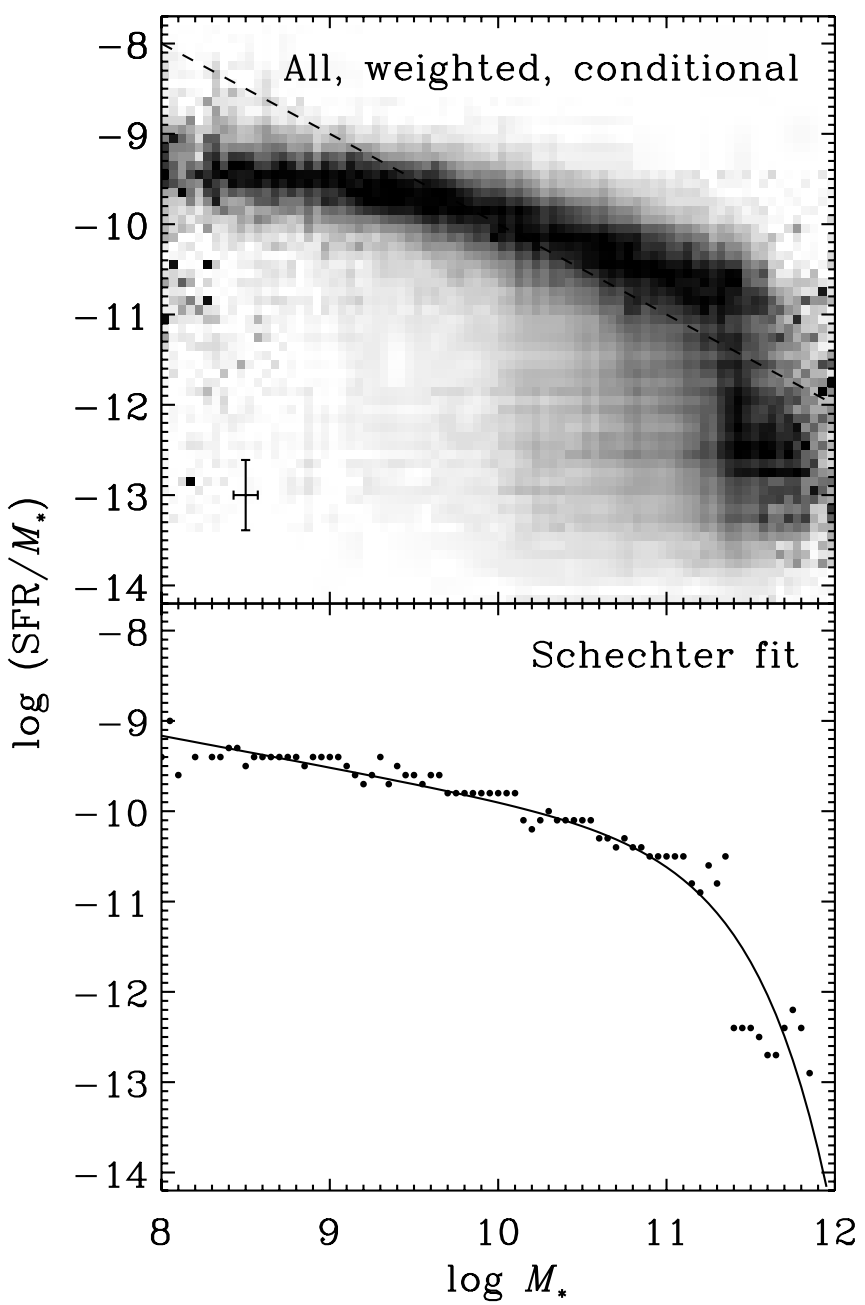

FIG. 16.-Conditional dependence of the star formation history on the stellar mass. Same sample as in Fig. 15, but with each 0.05 dex wide mass bin normalized to its maximum separately. This allows us to see what specific SFRs dominate at each mass, and to follow activity where there are to few objects to show in the standard plot. The bottom panel shows running modes from the top panel together with a Schechter-like fit to those points.

to some extent indicated in the CMDs (Fig. 1) but is more striking now.

The star-forming (SF) class, which forms a blue sequence in the CMD (Fig. 1), dominates the high values of the specific SFR. The sequence that was obvious in Figure 15 stands completely isolated here. In the weighted plot we see an ever-increasing density toward the lower mass, which reflects a rising low-mass slope of the mass function of late type (blue) galaxies. While the typical SFR rises from $0.1 M_{\odot} \mathrm{yr}^{-1}$ at the low-mass end to $10 M_{\odot} \mathrm{yr}^{-1}$ at $10^{11} M_{\odot}$ (consistent with the assumption that the more massive galaxies contain more gas and therefore have higher SFRs), the specific SFR actually declines by a factor of 10 . The tightness of the "star-forming" sequence (note that the formal error of the specific SFR is only 0.20 dex) represents an important indication that the galaxy's mass regulates the overall star formation history (see also Gavazzi et al. 1996; Boselli et al. 2001). It appears reasonable to assume that in the absence of an event that may disturb galaxy's gas reservoir, a galaxy would "naturally" sit on this tight sequence. This appears contrary to the suggestions that the red (non-SF) sequence forms by simple gas exhaustion, since we would then have a much wider range of (specific) SFRs at the given mass, for galaxies observed in the various stages of gas 


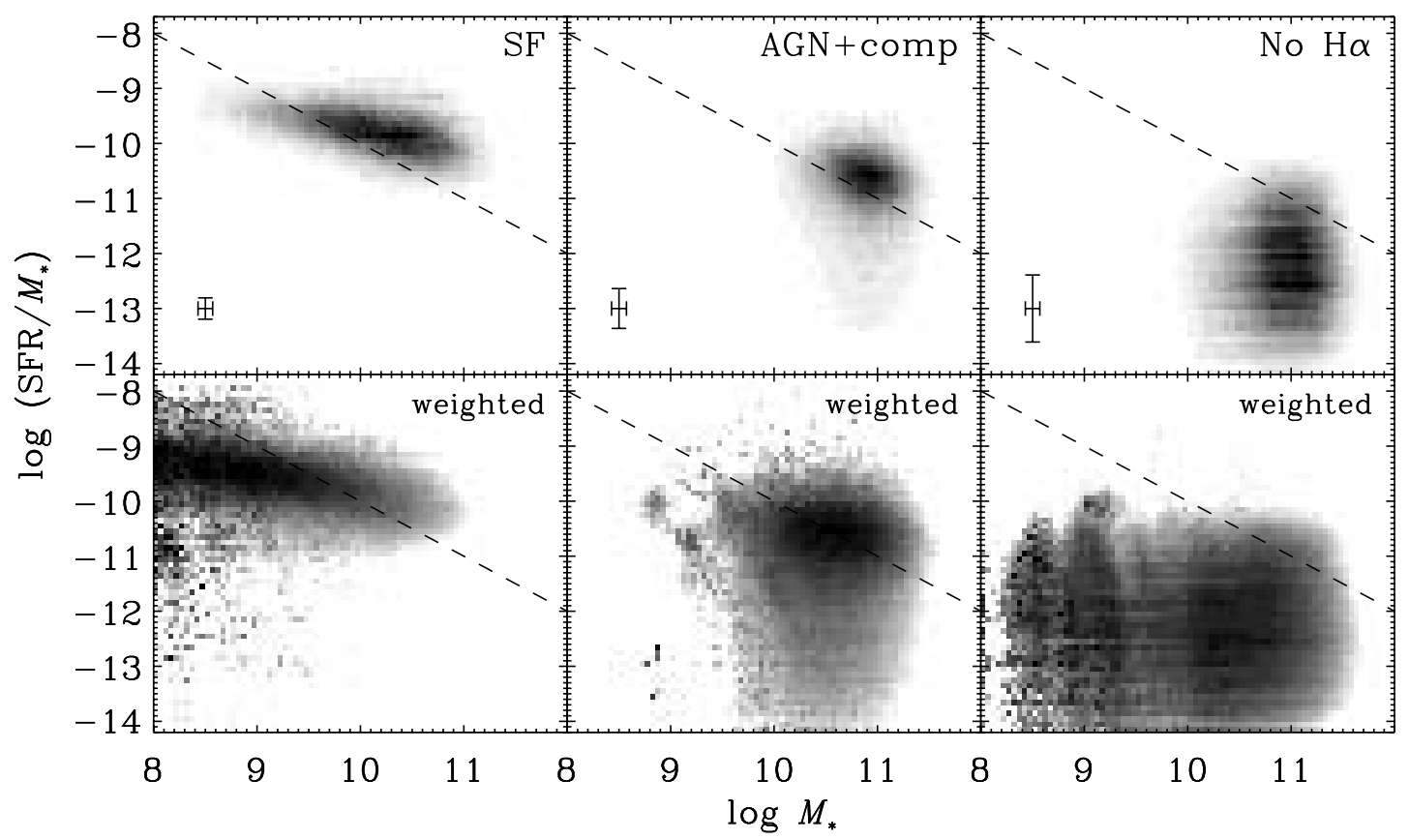

FIG. 17.-Dependence of the specific SFR on the stellar mass for different classes of galaxies. Star-forming (left panels); galaxies with AGNs (middle panels); and galaxies without $\mathrm{H} \alpha$ detection (right panels) all occupy distinct regions of the parameter space, indicating different SF histories. SF galaxies form a narrow sequence. AGNs have intermediate specific SFRs, and are predominantly high mass. Galaxies without $\mathrm{H} \alpha$, mostly red-sequence galaxies, have low specific SFRs. The dashed lines shows the reference SFR of $1 M_{\odot} \mathrm{yr}^{-1}$. The bottom panel shows values weighted by $V_{\max }$. Uneven behavior at low masses is because of small number of galaxies (or no galaxies) in those mass bins.

exhaustion. Note that for masses below $\log M_{*}=9.5$ we have an additional tail of high specific SFRs (best seen in volumeweighted plot), which is indicative of starbursts. We return to the width of the SF sequence in $\S 7.5$.

\subsection{Specific SFRs and Stellar Masses of Galaxies with an AGN}

We now turn to galaxies that lie on the AGN branch of the BPT diagram. These represent both galaxies classified as SF/AGN composites and "pure" AGNs (those lying in the region of the BPT diagram where non-SF ionizing source is necessary). We have already seen that a significant fraction of AGN hosts populates the green valley of the UV-to-optical CMD (Fig. 1) - the intermediate region between the blue and the red sequences. (However, note that in the optical CMD they would lie predominantly in the red sequence). A possible reason for the presence of these galaxies in the green valley is that they are entering the stage in which their star formation is being shut off, and the colors are becoming red as a result. Martin et al. (2007) using the GALEX data, has demonstrated that if the SF of galaxies lying in the green valley is indeed being quenched, then their color evolution implies a mass flux (transfer from blue to red) that roughly matches the mass increase required to populate the red sequence between the epochs $z \sim 1$ and 0 (Bell et al. 2004; Faber et al. 2007).

In the middle panels of Figure 17 we present specific SFR against mass for AGNs and the SF/AGN composites combined. The typical error of the specific SFR is 0.35 dex. Galaxies with AGNs populate the region between the SF galaxies with no AGNs and the galaxies with no $\mathrm{H} \alpha$ (adjacent panels), in a more striking manner than even in the UV-to-optical color-magnitude diagram. We note that these AGNs are of type 2 (Seyfert 2s and LINERs), which are not expected to affect the stellar continuum, especially not in the integrated galaxy light (Kauffmann et al. 2003b, and references therein). In galaxies having a luminous type $2 \mathrm{AGN}$, Kauffmann et al. (2003b) limit the AGN continuum contribution to less than a few percent in $r$ band. Assuming a power-law AGN continuum of the form $\propto \nu^{-1.5}$, this limit corresponds to less than $\sim 15 \%$ contribution in the UV. Another indication that the UV contribution from an AGN is low in the UV comes from the fact that the quality of our SED fitting for galaxies with AGNs is nearly the same as for the similar galaxies with no AGNs. Also, Kauffmann et al. (2007) explore GALEX imaging of a more nearby sample of AGN hosts and find that their UV emission is quite extended, thus unlikely to emanate from the central AGN.

The fact that most AGNs have intermediate star formation histories, and that this region is not occupied by galaxies unless they have an AGN, is remarkable in the light of recent suggestions that the AGN feedback may be regulating the star formation of massive galaxies (Springel et al. 2005). Still, we would like to know if there is a connection of galaxies hosting AGNs with normal star-forming galaxies with no AGNs. In Figure 18, we plot SF class (thin solid contours), and separate AGNs into "pure" AGNs (dashed contours) and SF/AGN composites (thick solid contours). Composites form the lower part of the AGN branch in the BPT diagram, and the "pure" AGNs the top part. Contours encompass $10 \%, 30 \%, 50 \%$, and $70 \%$ of each class's objects (i.e., their PDF densities), with no $V_{\max }$ weighting. We notice that composites "bridge" the normal SF population and that of "pure" AGNs; i.e., they have values of both the mass and the specific SFR that are intermediate between the two classes. AGNs, in addition, have a tail extending to very low values of the specific SFR. Another, more physically motivated way of splitting the AGN hosts is based on the strength (i.e., the accretion rate) of the AGN itself, as indicated by its [O III] $\lambda 5007 \AA$ luminosity. We use Kauffmann et al. (2003b) dust-corrected [O III] $\lambda 5007 \AA$ luminosities, and divide AGN hosts into strong and weak AGNs, using their demarcation of $L[\mathrm{O} \mathrm{III}]=10^{7} L_{\odot}$. We apply this division on all galaxies lying on the AGN branch of the BPT diagram (we refer the reader to Kauffmann et al. [2003b] for a visual presentation of where AGNs of various strengths lie on the BPT diagram). We show in Figure 19 the relation between the specific 


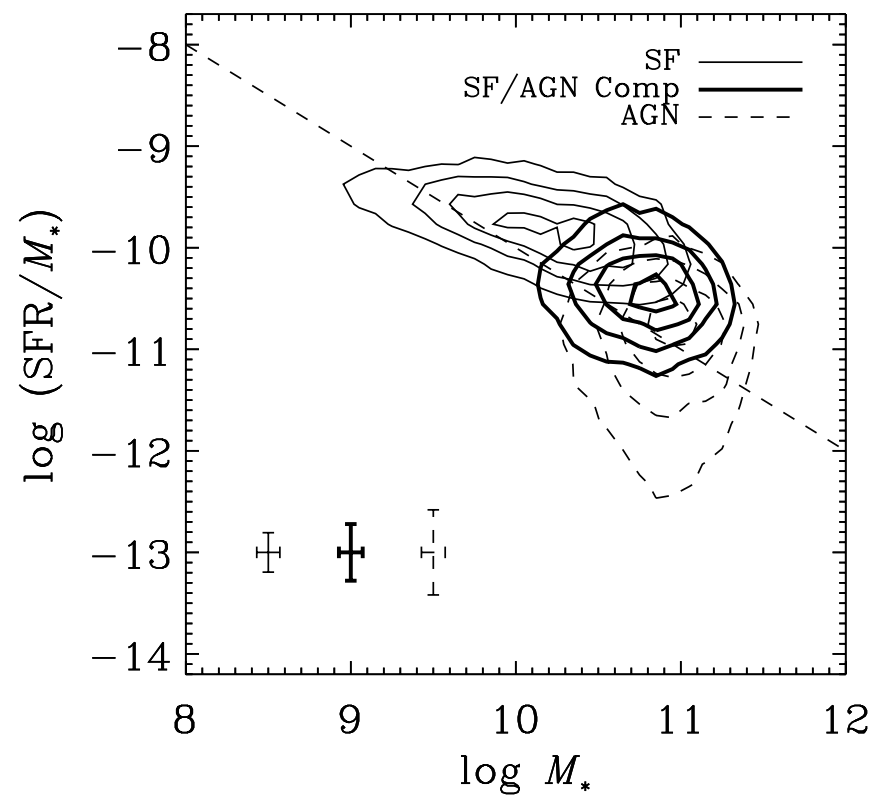

FIG. 18.- Specific SFR and mass of star forming, and of galaxies hosting AGNs. We plot density contours for normal SF galaxies having no AGNs (thin solid contours), of SF/AGN composites (bold solid contours), and of "pure" AGNs (dashed contours). The SF/AGN composite class (bottom part of the AGN branch in BPT diagram) bridges normal SF galaxies and the AGNs lying on the top part of the AGN branch. Contours of unweighted distribution encompass $10 \%$, $30 \%, 50 \%$, and $70 \%$ of objects, i.e., their composite PDF densities.

SFR and mass for SF galaxies (thin solid contours) and strong (thick solid contours) and weak (dashed contours) AGNs. Apparently, strong AGNs lie on the massive continuation of the SF sequence, with SFRs as high as those of the massive galaxies of the SF class (i.e., the offset from the dashed line is similar). Higher masses with similar SFRs makes their specific SFRs take more intermediate values. Weak AGNs fall at lower star formation rates relative to the strong AGNs, with a tail toward very low star formation rates that extends well into the domain of the quiescent galaxies (see below). Kauffmann et al. (2003b) using the optical spectroscopy, found that strong AGNs have younger populations than weak AGNs, which we qualitatively confirm, and provide a closer connection between strong AGNs and normal SF galaxies.

Here we propose an evolutionary progression for massive galaxies: from SF galaxies with no AGNs, to strong AGNs that are more massive yet with similar SFRs, to weak AGNs that have masses similar to strong AGNs but have lower (more suppressed) SFRs. Thus, the weak AGNs would represent a population with fading star formation. We emphasize that while most optical and structural properties of the majority of AGNs would place them in the optical red sequence, we find that they represent a smooth, massive extension of the star-forming sequence defined by optically blue galaxies. This picture is supplemented by the results of Kauffmann et al. (2007). They confirm that the majority of blue NUV $-r$ galaxies with high velocity dispersion host AGNs, and suggest that strong AGNs require extended gas-rich disks to provide a reservoir of gas that is fueled into an AGN. Previously, without the UV data, Kauffmann et al. (2003b) suggested that many type 2 AGNs are poststarburst systems. Such a possibility, by itself, allows at least two different evolutionary scenarios. One in which a normal red-sequence galaxy experiences a burst (say, through a minor gas-rich merger) that also fuels an AGN, and another in which the AGN is originally concurrent with normal SF. Our results, showing a smooth sequence that begins with SF galaxies without AGNs and extends at its massive end to AGN

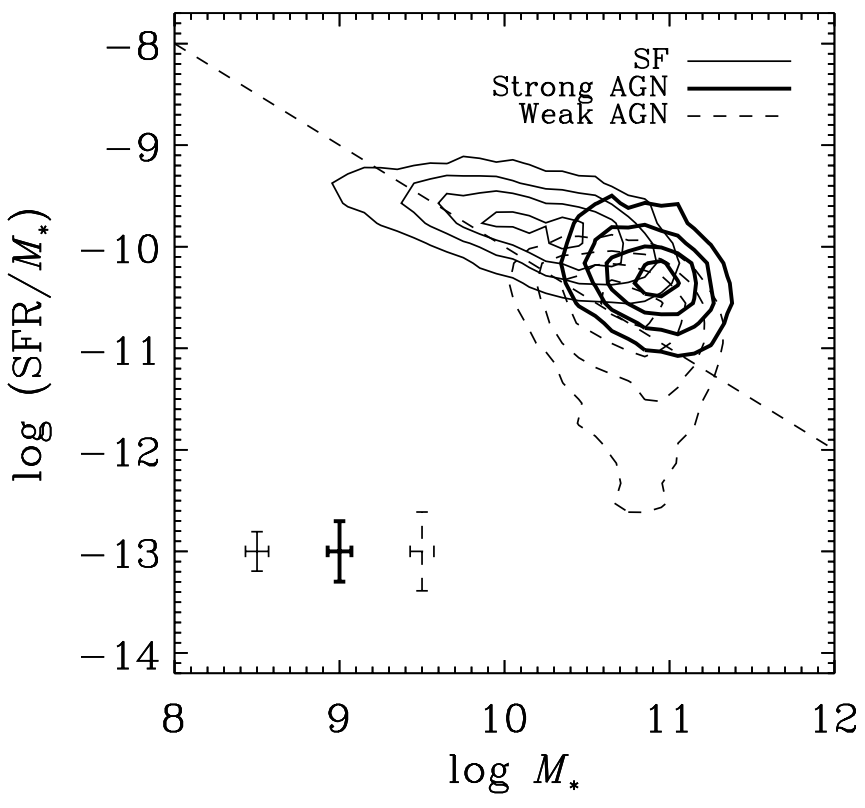

FIG. 19.- Specific SFR and mass of star forming, and of galaxies hosting AGNs of various strengths. We again show SF galaxies with no AGNs (thin solid contours), galaxies with strong AGNs (bold solid contours), and galaxies with weak AGNs (dashed contours). The strong/weak division of all galaxies on the AGN branch of the BPT diagram is based on the [O III] $25007 \AA$ A luminosity. Strong AGNs appear to form a continuation of the SF sequence, having similar SFRs, but larger masses. Weak AGNs have similar masses as strong AGNs but lower (specific) SFRs, with a tail extending to values typical of "quiescent" galaxies. Contours of unweighted distribution encompass $10 \%, 30 \%, 50 \%$, and $70 \%$ of objects, i.e., their composite PDF densities.

hosts with different levels of star formation, appear more compatible with the second scenario.

While neither Kauffmann et al. (2007) nor our study directly proves that AGNs are actually responsible for the subsequent quenching of the star formation, they do provide more evidence for the connection between the AGNs and the fading star formation in massive galaxies. Such picture is qualitatively compatible with recent theoretical work based on $N$-body simulations of galaxy evolution and semianalytic treatment of feedback. Specifically, the "radio"-mode AGN feedback, proposed by Croton et al. (2006) demonstrates that the galaxies situated in dark halos above some mass start to suppress the accretion of gas, and at the same time suppress further star formation. These are called "radio" AGNs because they can have black hole accretion rates well below Eddington, as is the case in our sample. Also, this mechanism is efficient in very massive galaxies. From the weighted plot of AGNs (Fig. 17; bottom middle panel) we find that the mass function of AGNs peaks at around $4 \times 10^{10} M_{\odot}$ and has an abrupt turnover at lower masses. This was noted by Kauffmann et al. (2003b), who also demonstrate that the low-mass cutoff is not likely to be a selection effect; if a low-mass galaxy contained anything but the very weakest AGN it would be classified as an AGN in the BPT diagram. The connection that GALEX has provided between the SF galaxies and the AGN feedback is quite reassuring, and evidently further work is required to complete this picture.

\subsection{Specific SFRs and Stellar Masses of Galaxies with no $\mathrm{H} \alpha$ Emission}

Finally, we concentrate on galaxies for which no $\mathrm{H} \alpha$ emission was detected in SDSS spectra. These galaxies have red optical colors, which puts them in the "red sequence" category. These 
galaxies are often referred to as "old, red, and dead," because of the presumably old populations and the lack of current SF. However, in the UV to optical colors (Fig. 1) we saw that some of these galaxies have relatively blue colors (which indicates that one needs to be careful when using the term such as "red sequence," since it apparently depends on the color used). In the right-hand panels of Figure 17 we show specific SFRs of galaxies with no $\mathrm{H} \alpha$. In the unweighted plot they occupy a region of the parameter space distinct from the star-forming and AGN galaxies, with specific SFRs typically lower than for those two classes. The volume-corrected plot still puts the bulk of them lower than the other two classes, but the overlap with the low specific-SFR tail of the AGN is more prominent. Also, the mass extent is now displayed accurately, with the mass function turning over and flattening at lower masses.

If we expect most red-sequence galaxies to have no star formation at all, how do we interpret the (specific) SFRs derived here? First, we note that the specific SFRs (and also just the SFRs) are more poorly constrained than for any other class (average formal error in specific SFR is 0.63 dex). This should be interpreted in a way that the observed SEDs are in many cases compatible with a very wide range of SFRs, mostly very low ones. Let us note that our model SF histories do not contain truncated models, but only exponentially declining models (with added starbursts), which can never reach exact zero SFR. The dynamic range of SFR in models is determined by the range of exponential timescales, which in our case have a minimal $e$-folding time of $1 \mathrm{Gyr}$. This produces a lower limit of $\log (\mathrm{SFR} / L) \approx-14$, which is roughly equivalent to the lower limit in the specific SFR shown in Figure 17. While many galaxies in this class are compatible with this lower limit, they do not cluster close to it, because in the absence of stronger constraints, higher SFRs will also be allowed (i.e., to firmly constrain that the SFR is, say, smaller than $0.001 M_{\odot} \mathrm{yr}^{-1}$ one needs to measure a very low amount of UV light with a very high precision). However, there are clear cases in which a galaxy classified as having no $\mathrm{H} \alpha$ has a relatively well constrained SFR. For some of them it is as high as $1 M_{\odot} \mathrm{yr}^{-1}$ (crossing the dashed line). What is the morphology of galaxies with high (specific) SFRs, yet no $\mathrm{H} \alpha$ emission? There are 837 galaxies with $\log M_{*}>10$ and $\log \left(\mathrm{SFR} / M_{*}\right)>-11$. We inspect SDSS images of these galaxies. Most appear as normal early-type galaxies (with several obvious E+A types), which was already indicated in Rich et al. (2005), who find that some morphologically and spectroscopically early-type galaxies show blue UV-to-optical colors. However, one quarter shows some sign of structure or a disturbed light profile. This fraction would very likely be higher in deeper images or images with higher spatial resolution. One quarter of galaxies with structure show traces of spiral arms. In most cases these spiral arms are faint, and possibly represent cases in which the star formation was recently shut down (leading to the lack of emission lines), yet the residual UV light and the spiral structure remain. Such galaxies have been recognized in galaxy clusters as "passive spirals" (van den Bergh 1976; Poggianti et al. 1999) and are believed to represent a transition between a spiral and an S0 galaxy. In the inspected sample these galaxies are found at all redshifts, indicating that the reason $\mathrm{H} \alpha$ is not detected is most likely not due to the aperture size being smaller than the size of the galaxy from which the integral flux is measured (especially for more nearby galaxies). Many of the galaxies with structure exhibit rings - from very faint ones to bright blue ones. In some of those cases SF could be occurring outside of the spectroscopic fiber, leading to nondetection of $\mathrm{H} \alpha$.

One can be concerned that our models attribute the UV light from old populations (the UV "upturn") to young stars, thus im- plying an artificially high star formation rate. Bruzual \& Charlot (2003) models do include post-AGB phases, but not the extreme blue horizontal branch, which is believed (by no means unanimously) to be responsible for the UV upturn in early-type galaxies (Brown et al. 2000). However, Donas et al. (2007) explore nearby "pure" elliptical galaxies and find that both the UV and the optical colors that they observe are fully reproduced in the models that we use in this work, and that if the UV excess from the actual star formation is present, it is discernible from the combination of UV and optical colors. Therefore, our models do appear to account for UV light from old populations and therefore some of the objects with no $\mathrm{H} \alpha$ emission are quite likely to have had actual recent SF. This conclusion is also reached in other studies based on GALEX data (Yi et al. 2005; Kaviraj et al. 2007; Schawinski et al. 2007), where the blue UV-to-optical colors of morphologically selected early-type galaxies are interpreted as signs of recent star formation. It is quite conceivable that these galaxies are in a poststarburst phase, so that the $\mathrm{H} \alpha$ emission is no longer present, while the traces of star formation are still visible in the UV. In evolutionary terms, as in the case of AGNs, a question remains whether this recent $\mathrm{SF}$ is the sign of a previously normal SF that is now quenched, or is it the result of a small SF episode in an already quiescent galaxy. In the latter case, the starburst could be due to a minor merger with a gas-rich galaxy. Most likely both processes are present, and the forthcoming work will address the relative importance of each.

\subsection{Relationships between Specific SFR and Mass}

One would like to quantify the trends of the specific SFR trends with respect to mass in order to facilitate easier comparison of our optically selected, local-universe sample to studies at other redshifts, or samples selected in a different manner (keeping in mind that a conversion may be required for a different choice of IMF or $H_{0}$ ).

If one can identify "pure" star-forming galaxies (with no AGN contribution) using the BPT diagram, then the star-forming sequence is best described with a linear fit:

$$
\log \left(\mathrm{SFR} / M_{*}\right)=-0.35\left(\log M_{*}-10\right)-9.83 .
$$

In many instances one does not have all the requisite spectral lines to make an exact classification, but instead relies on color to separate star-forming galaxies. Adopting a color cut of NUV $r=4$, which passes in between the blue and the red sequence, and taking galaxies on the blue side, we encompass all "pure" starforming galaxies, but also many SF/AGN composites (see Fig. 1). In that case, the star-forming sequence is better described with a piecewise linear fit:

$$
\begin{aligned}
& \log \left(\mathrm{SFR} / M_{*}\right)= \\
& \begin{cases}-0.17\left(\log M_{*}-10\right)-9.65, & \log M_{*} \leq 9.4, \\
-0.53\left(\log M_{*}-10\right)-9.87, & \log M_{*}>9.4 .\end{cases}
\end{aligned}
$$

This relation differs from equation (11) mostly because the high-mass end is somewhat lower-a consequence of including SF/AGN composites, which have lower specific SFRs than pure SF galaxies of the same mass. In both cases we have performed least-square fitting to the conditional volume-corrected composite PDFs (analogous to Fig. 16, top), binned into running modes in the $8.1<\log M_{*}<11.7$ range for SF galaxies, and the $8.0<$ $\log M_{*}<11.6$ range for blue galaxies.

The trend of the specific SFR with respect to mass for the entire sample is best observed in Figure 16 . We find that the 
parameterization having the form similar to Schechter (1976) luminosity function provides an excellent overall fit to this trend. Recently, Feulner et al. (2006) have used Schechter functions to describe the upper envelopes of their SFR $/ M_{*}$ vs. $M_{*}$ data points, but here we find that it represents a good description of the main trend. We use the Schechter function of the form

$$
\begin{aligned}
& \mathrm{SFR} / M_{*}= \\
& \left(\mathrm{SFR} / M_{*}\right)_{0} 10^{(\alpha+1)\left(\log M_{*}-\log M_{0}\right)} \exp \left(-10^{\left(\log M_{*}-\log M_{0}\right)}\right)
\end{aligned}
$$

where $\left(\mathrm{SFR} / M_{*}\right)_{0}, M_{0}$, and $\alpha$ describe the normalization, characteristic stellar mass, and the low-mass slope, respectively. Taking modes of the conditional distribution in the $8.0<\log M_{*}<$ 11.9 range, we find the following best-fitting parameters:

$$
\begin{gathered}
\left(\mathrm{SFR} / M_{*}\right)_{0}=5.96 \times 10^{-11} \mathrm{yr}^{-1}, \\
\log M_{0}=11.03, \quad \alpha=-1.35 .
\end{gathered}
$$

This fit, and the data points that were used to construct it are shown in the bottom panel of Figure 16.

In the paradigm of "downsizing," at higher redshifts the SF was more active in galaxies with larger mass. This should be reflected in the relative flattening of the SF sequence at higher redshifts. While most current studies observe an overall shift toward higher specific SFRs, the slope does not appear to change much (e.g., Noeske et al. 2007; Papovich et al. 2006). Our quantification of the specific SFR should enable more direct comparison with the local universe. In many cases, simple overplotting of equations (13) and (14) to other sets of data would be indicative (unless data are color-selected to include only the blue sequence, in which case we recommend using eq. [12]).

One often wishes to determine the average specific SFR of their sample. The average specific SFR can be defined in several ways, but the most used definition is one in which the total SFR in a volume-limited sample is simply divided by the total stellar mass. We call this the cosmic specific SFR:

$$
\mathrm{SFR} / M_{*}(\text { cosmic })=\frac{\sum\left({ }^{0.1} \mathrm{SFR} / V_{\max }\right)}{\sum\left(M_{*} / V_{\max }\right)},
$$

where ${ }^{0.1} \mathrm{SFR}$ is the SFR corrected to $z=0.1$ (see $\oint 8$ ). From our sample we find SFR $/ M_{*}($ cosmic $)=6.8 \times 10^{-11} \mathrm{yr}^{-1}$, which is in very good agreement with the equivalent measure in B04. How much is the estimate of the cosmic specific SFR dependent on the limiting mass of the sample? In Figure 20 we plot cosmic specific SFR as it would have been determined had we had a mass limit (and assuming the same magnitude limits). The right-hand $y$-axis shows a fraction of the "actual" (no mass limit) cosmic $\mathrm{SFR} / M_{*}$ at the given mass limit.

Finally, we ask with what precision can one use the relations to "predict" specific SFR from stellar mass? For blue galaxies, we find the average scatter around the mean sequence to be $0.5 \mathrm{dex}$. This result is only slightly affected after taking out in quadrature the formal measurement error of $0.2 \mathrm{dex}$. Therefore, the sequence has an intrinsic width, and is likely to depend on other factors besides the mass. The width itself is a function of mass. It declines at the rate of $-0.11 \mathrm{dex}^{-1}$; i.e., the scatter of the specific SFR at $\log M_{*}=8.0$ is $65 \%$ larger than at $\log M_{*}=10.5$. This is an indication that the star formation becomes less stochastic as the mass increases, which is what one expects considering that massive galaxies have a larger number of star-forming regions than dwarf galaxies.

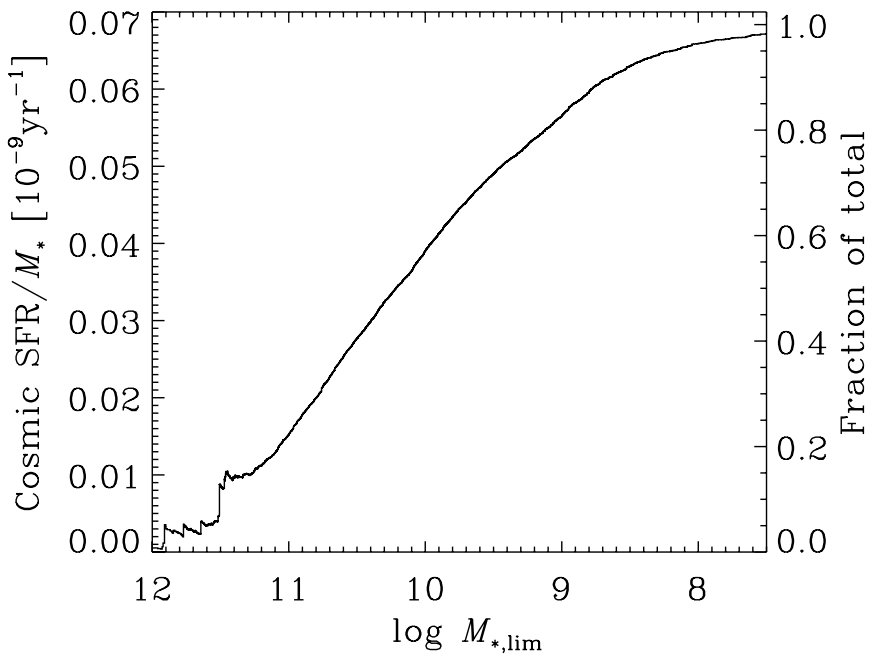

FIG. 20.-Cosmic specific SFR as a function of the limiting mass. We define cosmic specific SFR as the ratio of total SFR to total mass, each weighted by $V_{\max }$. The figure shows the effect of the limiting mass on the estimate of the cosmic specific SFR. SFRs have been corrected to $z=0.1$ by applying an evolution correction of the form $\propto(1+z)^{2.5}$.

\section{GLOBAL STAR FORMATION DENSITY}

In this section we use our SFRs to determine the global star formation density at $z=0.1$, the mean redshift of our sample. In order to take into account that the sample has a spread of redshifts, and therefore probes a range of cosmic star formation history, in the remainder of the section each galaxy's SFR estimate is "corrected" to the mean redshift of the survey, where the uncertainties in the correction are minimized. To make the correction, one assumes a cosmic star formation density evolution of the form $(1+z)^{\beta}$, where B04 take $\beta=3$. Therefore, the corrected SFR for a galaxy lying at redshift $z$ is ${ }^{0.1} \mathrm{SFR}=[1.1 /(1+z)]^{\beta} \mathrm{SFR}$.

Since we will be comparing our results to those obtained by B04, we first describe their methodology and apply it to our data. B04 obtain star formation density ( $\left.\rho_{\mathrm{SFR}}\right)$, and its formal (random) error, by Monte Carlo method applied to SFR PDFs in the following way. First, 100 different bootstrap samples are constructed from the original sample, using drawings with replacements. Bootstrap samples have the same number of objects as the original sample. A random number is then used to select a value from the cumulative PDFs for each galaxy in the bootstrap sample. The random sampling of the PDF is repeated 30 times. Each SFR PDF is weighted by $1 / V_{\max }$ and summed to get $\rho_{\mathrm{SFR}}$. From the ensemble of such samplings the mode and the $16 \%$ and $84 \%$ confidence levels are determined for the entire sample, and for each galaxy class. The results of this exercise are presented in Table 3 of B 04 . The random error of the total $\rho_{\mathrm{SFR}}$ is found to be around 0.018 in units of $10^{-2} h_{70} M_{\odot} \mathrm{yr}^{-1} \mathrm{Mpc}^{-3}$. For our sample, we perform only the multiple probing of each galaxy's cumulative PDF, without bootstrapping the sample. Performing 100 Monte Carlo summations, we find a random error of the total $\rho_{\mathrm{SFR}}$ to be 0.017 , in the same units. Therefore, the two studies have comparable formal random errors, which is not surprising given the fact that the formal errors of the SFRs are comparable. In any case, these random errors are smaller than some systematic uncertainties that we will explore below.

How do the actual values of SFR density compare? First, we notice that the B04 method is essentially equivalent to the summation of the medians of the PDFs since the average value of a random number that probes the cumulative PDF is 0.5 . In Table 2 we compare the values obtained from the summation of the 
TABLE 2

Comparison of SFR Densities at $z=0.1$ in this Study and in B04, Derived using Compatible Methodology

\begin{tabular}{|c|c|c|c|c|c|}
\hline Class & $\rho_{\mathrm{SFR}}($ This Paper) & $\%$ of Total & $\rho_{\mathrm{SFR}}(\mathrm{B} 04)$ & $\%$ of Total & Fractional Change \\
\hline (2) & 1.953 & 100.0 & 1.980 & 100.0 & 0.99 \\
\hline SF & 1.395 & 71.4 & 1.013 & 51.2 & 1.38 \\
\hline SF $($ low $S / N) \ldots \ldots \ldots \ldots \ldots \ldots$ & 0.296 & 15.2 & 0.455 & 23.0 & 0.65 \\
\hline Comp & 0.147 & 7.5 & 0.207 & 10.5 & 0.71 \\
\hline AGN & 0.076 & 3.9 & 0.084 & 4.2 & 0.90 \\
\hline
\end{tabular}

Notes.-For this comparison we adopt $\beta=3$ SFR density evolution. SFR density is given in units of $10^{-2} h_{70} M_{\odot} \mathrm{yr}^{-1} \mathrm{Mpc}^{-3}$.

medians of our SFR PDFs, to the average of the 16th and 84th percentiles of B04 values (which should be close to the median). Our estimate includes the correction for galaxies excluded from the sample because of the poor SED fits ( $\$ 4.2$ ). These corrections are $1.6 \%-2.8 \%$, depending on the class. Looking at the first row, we see that our estimate of the total SFR density is very close to that of B04. However, this is a mere coincidence when we look at how the densities compare by class. Our SFR density for the SF class is $38 \%$ higher; however, for all other classes the estimate is lower-between $10 \%$ less for AGNs to 5.7 times less for galaxies without $\mathrm{H} \alpha$. The causes for these differences can be traced from the analysis of SFRs in $\S 5$. Consequently, the contribution of each class to the total SFR density has changed as well. SF class now contributes some $70 \%$, while it was around $50 \%$ in B04. This comes at the expense of other classes (except for the AGNs), and most significantly from much lower contribution of galaxies without $\mathrm{H} \alpha(2 \%$, vs. $11 \%$ in $\mathrm{B} 04)$.

We next describe the calculation of our SFR density, which somewhat differs from B04. Direct summation that was used to find SFR density in B04 assumes that the losses from the incompleteness of the sample are insignificant. B04 estimates them to be between $1 \%$ and $2 \%$. We fit a Schechter fit to the number density of galaxies of various classes and integrate the luminosity density down to our completeness limit of $M_{r}=-14.75$, and to a limit that is 10 mag fainter. We compare the two total luminosity densities and find that $1.1 \%$ of luminosity density is below our limit for SF (and low-S/N SF) galaxies. For other classes, the loss is practically zero. Therefore, for our SFR density estimate we perform the direct summation by taking galaxies $M_{r}<-14.75$, and then correcting the SFR density. This result differs insignificantly from one in which we would sum all galaxies with no magnitude limit. Our SFR density estimates are obtained by summing the averages of the SFR estimates. We prefer summing averages over medians because it is equivalent to summing an entire PDF. Averages produce a result that is 5\% higher than the medians. Also, we adopt the exponent of the SFR density evolution

TABLE 3

SFR Densities at $z=0.1$ Determined in This Study

\begin{tabular}{|c|c|c|}
\hline Class & $\rho_{\mathrm{SFR}}$ & $\%$ of Total \\
\hline 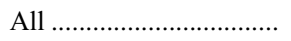 & 1.828 & 100.0 \\
\hline . & 1.328 & 72.6 \\
\hline $\mathrm{SF}($ low $\mathrm{S} / \mathrm{N}) \ldots \ldots \ldots \ldots \ldots$ & 0.277 & 15.2 \\
\hline 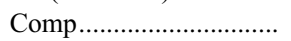 & 0.136 & 7.5 \\
\hline 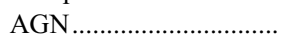 & 0.067 & 3.6 \\
\hline No $\mathrm{H} \alpha$ & 0.021 & 1.1 \\
\hline
\end{tabular}

Notes.-We adopt $\beta=2.5$ SFR density evolution. SFR density is given in units of $10^{-2} h_{70} M_{\odot} \mathrm{yr}^{-1} \mathrm{Mpc}^{-3}$. Random error of SFR density for "All” galaxies is 0.017 in same units. See text for the discussion of systematic errors. $\beta=2.5 \pm 0.7$, determined by Schiminovich et al. (2005) from GALEX-VVDS measurements.

The results, and the breakdown by class, are presented in Table 3 and shown in Figure 21. These are our nominal SFR densities. Galaxies for which no classification was possible (see $\S 3.2$ ) are included in the SF class. They constitute $0.8 \%$ of the total SFR density. Altogether, $88 \%$ of star formation occurs in "normal" SF galaxies, and another $11 \%$ in galaxies that host an AGN. Up to $1 \%$ occurs in galaxies that are quiescent based on the optical spectroscopy.

The uncertainty in the evolution exponent leads to the systematic uncertainty in the total SFR density of $2 \%$. By far the largest uncertainty stems from the systematic uncertainties in the determination of the dust attenuation, and hence the SFR of SF galaxies (§ 5.4). We estimate the degree of this error by simply replacing the SFRs of SF galaxies by those obtained by B04. This error is preferentially in the direction that the " $\mathrm{H} \alpha$ " estimate becomes $11 \%$ higher for the SF class, leading to the total density that is $8 \%$ higher. We also take into account that our SFR estimates are averaged over $10^{8} \mathrm{yr}$. Using $10^{7} \mathrm{yr}$ instead, we obtain a SFR density that is $1 \%$ lower, which we will treat as the systematic error. Finally, another small source of systematic error comes from the error in the estimate of survey area of $0.3 \%$. Adding up in quadrature random and systematic errors in the positive and in the negative directions, we arrive at our total, dust-corrected SFR density estimate at $z=0.1$ :

$$
\rho_{\mathrm{SFR}}=1.828_{-0.039}^{+0.148} \times 10^{-2} h_{70} M_{\odot} \mathrm{yr}^{-1} \mathrm{Mpc}^{-3},
$$

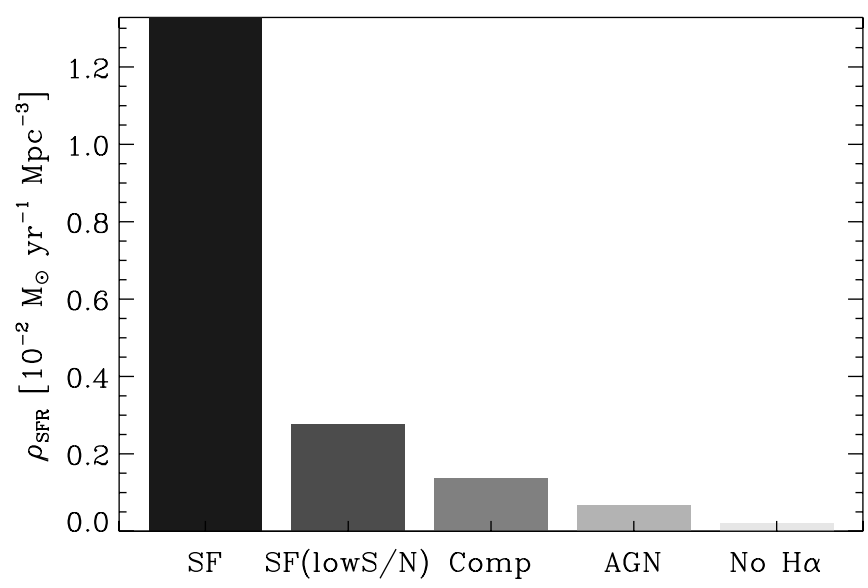

FIG. 21.-Total star formation rate densities for different classes of galaxies. $88 \%$ of total star formation occurs in "normal" SF galaxies (including low-S/N SF class), and another $11 \%$ in galaxies that host an AGN. Up to $1 \%$ occurs in galaxies that are quiescent based on the optical spectroscopy (no $\mathrm{H} \alpha$ class). Estimates are given for the mean redshift of the sample of $z=0.1$, by applying an evolution correction of the form $\propto(1+z)^{2.5}$. 


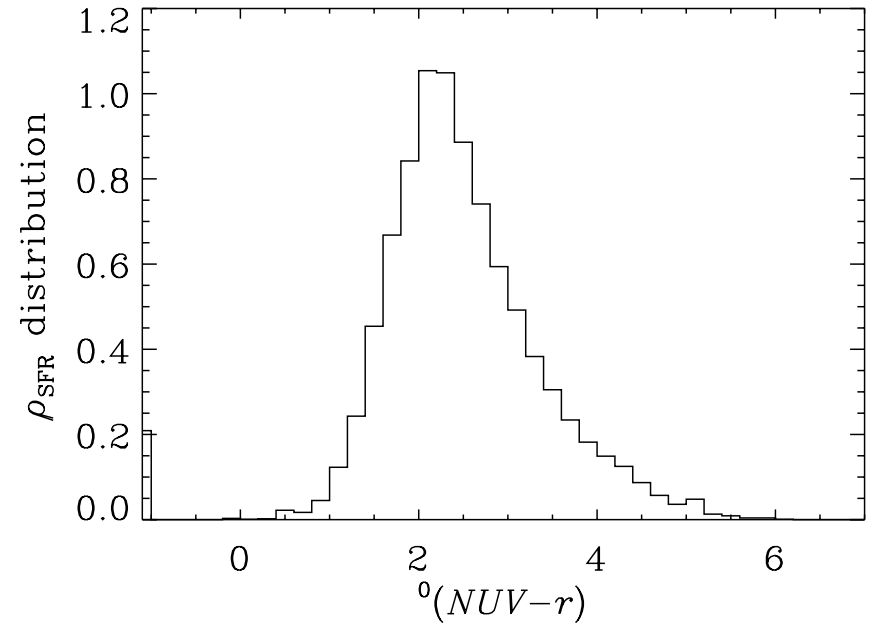

Fig. 22.-Distribution of the star formation density (at $z=0.1$ ) as a function of color. We choose UV-to-optical color, which shows a distinct bimodality in the number density of galaxies. The SFR density distribution is unimodal, and peaks sharply. There is a tail toward the red galaxies, which is in part due to the intrinsic dust reddening. A large fraction (94\%) of the star formation density arises from galaxies blueward of NUV $-r=4-$ our nominal division between the blue and red galaxies. First bins includes all objects without NUV detection. Objects below our absolute magnitude completeness limit ( $\sim 1 \%$ of SFR density) are not included. The units of SFR density distribution are $10^{-2} h_{70} M_{\odot} \mathrm{yr}^{-1} \mathrm{Mpc}^{-3} \mathrm{mag}^{-1}$.

which assumes the Chabrier IMF. For a compilation of recent measurements of the local $\rho_{\mathrm{SFR}}$ please refer to Hanish et al. (2006). Our $\rho_{\text {SFR }}$ agrees very well $(<1 \sigma)$ with four of the six estimates listed in their Table 1, but has significantly smaller error bars. Note that except for B04, all other estimates are based on samples with fewer than 300 galaxies, and many apply fixed dust correction for all galaxies. We can find the average FUV attenuation by "de-correcting" our SFRs by adding our FUV attenuation estimates to each galaxy. Such uncorrected SFR density is smaller by a factor of 6.34. In other words, the average FUV attenuation for the volume-complete sample is $A_{\mathrm{FUV}}=2.01 \mathrm{mag}$.

We next explore the distribution of global SFR density across the galaxies having different properties. In Figure 22 we show the distribution of SFR density against the rest-frame NUV $-r$ color. The distribution sharply peaks at ${ }^{0}(\mathrm{NUV}-r)=2.2$. While the number density distribution is strikingly bimodal in this color, the SFR distribution is very even. The blue edge is more abrupt then the red side, which shows a tail extending to redsequence colors, in part due to the intrinsic dust reddening. We have previously used an NUV $-r=4$ cut, which goes across the green valley, to conveniently divide the blue and the red sequences. From Figure 22 we see that the large fraction (94\%) of star formation occurs blue of this cut. The first bin sums SFR density in galaxies lacking $>3 \sigma$ measurement in NUV ( $2 \%$ of total).

UV to optical color is easy to measure, but gives only a very rough idea of a galaxy's SF history. Therefore, in Figure 23 we plot the SF density distribution against the specific SFR. The picture is somewhat different than from the distribution against the color. First, the high specific-SFR end does not fall as evenly as did the blue NUV $-r$ color end; instead, we see an excess of galaxies with $\log \left(\mathrm{SFR} / M_{*}\right) \approx-8.3$. These are the bursty, lowmass galaxies mentioned in $\S 7.2$. The reason that NUV $-r$ color does not distinguish this population is that it "saturates" for galaxies with $\log \left(\mathrm{SFR} / M_{*}\right) \approx-9$, so that all galaxies with higher specific SFR will have similar NUV $-r$ colors. FUV (included in the specific SFR) is needed to break the degeneracy. On the side of the low specific SFR (equivalent to redder NUV $-r$ colors), the decline is more steep than for the colors. The difference arises

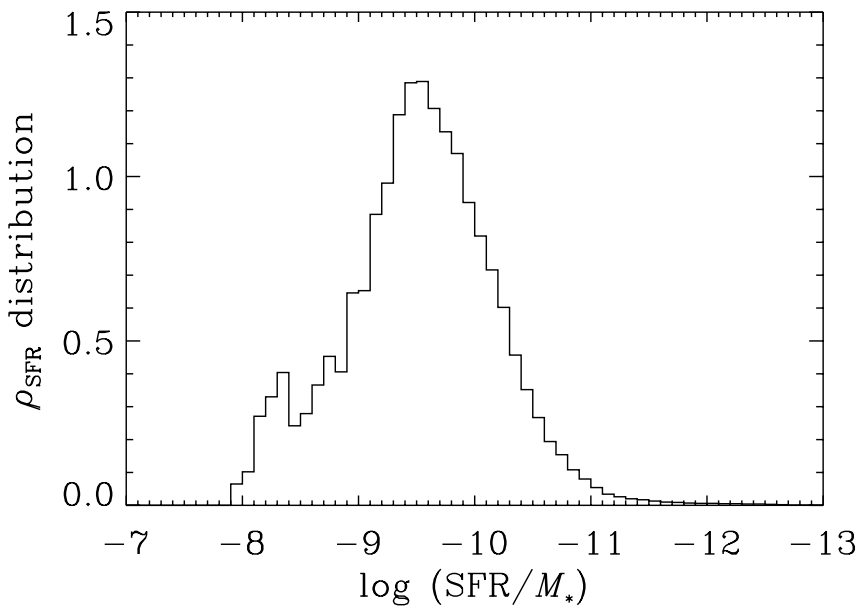

Fig. 23.-Distribution of the star formation density (at $z=0.1$ ) in galaxies with different specific SFRs. Specific SFR is an indicator of a galaxy's star formation history. The distribution shows a "bump" at the high end due to the population of bursty, low-mass galaxies. There is a steep decline toward the galaxies with low specific SFR. The units of SFR density distribution are $10^{-2} h_{70} M_{\odot} \mathrm{yr}^{-1} \mathrm{Mpc}^{-3} \mathrm{dex}^{-1}$.

from the fact that the specific SFR, unlike NUV $-r$ color, does take out the effects of the intrinsic dust attenuation.

Finally, in Figure 24 we show the distribution of SFR density across the galaxies with different stellar masses. The fraction of SF occurring in galaxies with increasing mass rises almost linearly. The peak of the SF today occurs in galaxies having a mass of $\approx 4 \times 10^{10} M_{\odot}$. This mass is close to the "transitional" mass of Kauffmann et al. (2003a), which is effectively a mass at which blue and red mass functions cross; i.e., the mass above which the red galaxies become more numerous. In the paradigm of downsizing, we would expect this distribution to move toward the higher masses at higher redshifts. This, however, is often difficult to establish since the completeness limits of many high-redshift surveys are uncomfortably close to the peak of the distribution whose position one tries to establish. In Figure 25 we show the cumulative SFR density as a function of stellar mass, summing up from the highest mass galaxies. This figure shows how much SF is missed (in the local universe) by being limited to a given stellar

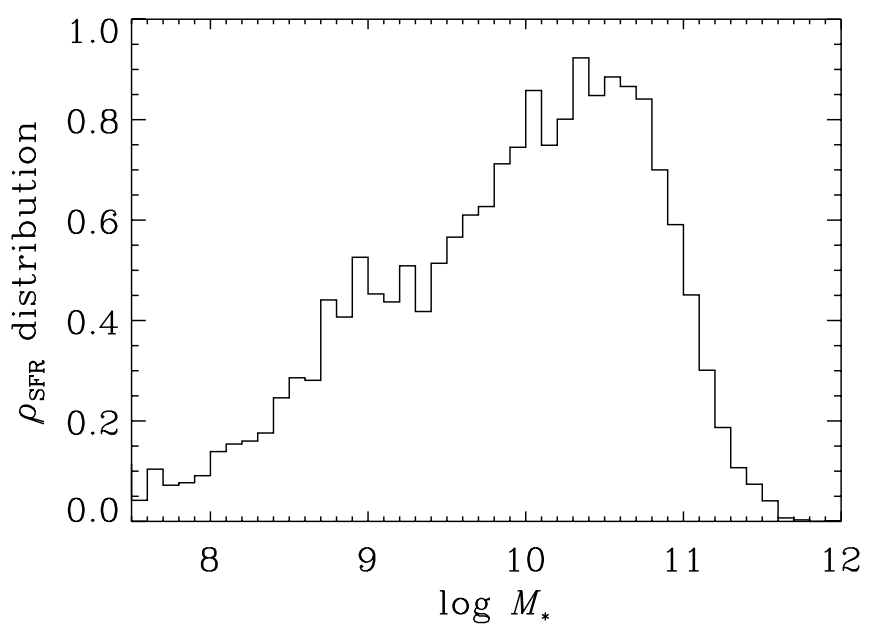

FIG. 24.-Distribution of the star formation density (at $z=0.1$ ) in galaxies of different stellar mass. The fractional star formation density increases with galaxy's stellar mass and reaches a maximum at $\approx 4 \times 10^{10} M_{\odot}$, which is close to the "transitional" mass of Kauffmann et al. (2003a). Our sample is incomplete at $\log M_{*}<$ 7.5. The units of SFR density distribution are $10^{-2} h_{70} M_{\odot} \mathrm{yr}^{-1} \mathrm{Mpc}^{-3} \mathrm{dex}^{-1}$. 


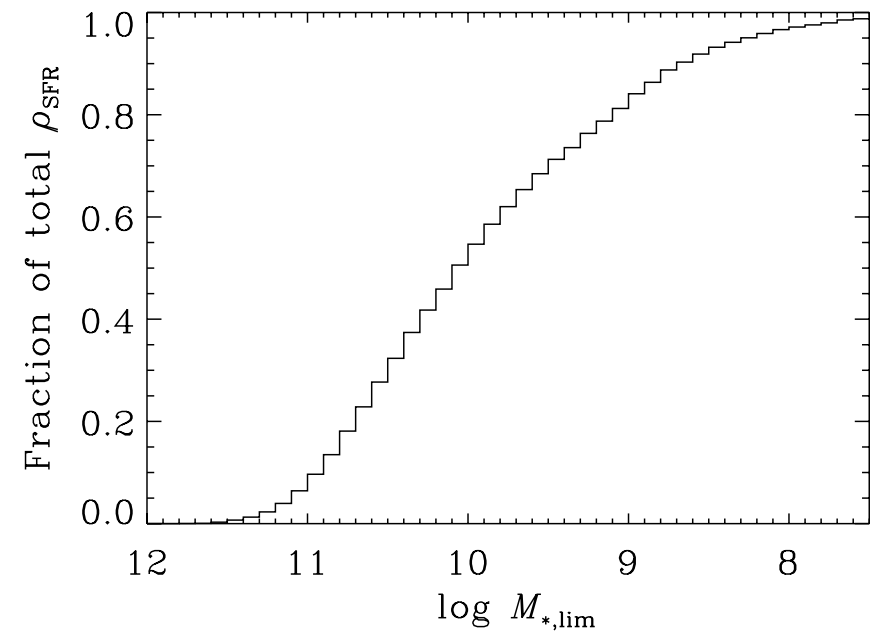

FIG. 25.-Fraction of the total star formation density (at $z=0.1$ ) as a function of limiting stellar mass. The summing is performed from the high-mass end. Therefore, at each mass, the plot shows how much SF is missed by not including less massive galaxies. Our sample is incomplete at $\log M_{*}<7.5$, where we estimate $1 \%$ of SF takes place.

mass (and assuming the same magnitude limits). Thus, a limit of $10^{11} M_{\odot}$ captures only $6 \%$ of the total SFR density. In order to reach $50 \%$, one needs to probe galaxies more massive than $10^{10}$ $M_{\odot}$. Today, $1 / 2$ of the total SFR density comes from galaxies in the mass range $9.3<\log M_{*}<10.6$.

\section{CONCLUSIONS}

We have considered the measurement of the star formation rate using a sample of 50,000 optically selected galaxies with GALEX and SDSS photometry and SDSS redshifts. We have given a detailed description of our approach to SED fitting, including the use of a large library of population models that include a physically motivated treatment of dust attenuation. These give dust-corrected SFRs ("UV" SFRs) of galaxies in the local universe. We show that there are no inherent problems that would prevent UV from serving as a reliable SFR indicator, despite past concerns over population synthesis and dust attenuation models. We recite some of the highlights from our study.

1. 25 minute imaging from GALEX in two UV bands can be used to derive SFRs with a formal accuracy of 0.2 dex for $r<18$ mag star-forming galaxies with known redshifts.

2. For galaxies for which the dominant ionizing source are young stars (as determined using $3^{\prime \prime}$ fiber measurements and the BPT diagram), the SFRs obtained through SED fitting ("UV" SFRs) agree exceptionally well with dust-corrected SFRs obtained from modeling of the nebular lines-essentially $\mathrm{H} \alpha$, corrected using a Balmer decrement ("H $\alpha$ " SFRs), as long as the same dust model is applied to both methods. The comparison presented here is at a scale that is 2 to 3 orders of magnitude larger than the comparison of these SF indicators done in the past.

3. The Charlot \& Fall (2000) dust attenuation model that we apply to "UV" SFRs and that B04 apply to "H $\alpha$ " SFRs succeeds in producing $\mathrm{UV}$ and $\mathrm{H} \alpha$ dust attenuation estimates that lead to compatible SFRs.

4. Minor differences between the "UV" and "H $\alpha$ " SFRs exist at the extremes of galaxy stellar mass distributions (equivalent to the extremes of the dust attenuations). They arise either from somewhat weaker constraints from the SED-derived dust attenuation, or from the mass-dependent deviations of the assumed $\propto \lambda^{-0.7}$ extinction law in the UV regime.
5. If the systematics in the attenuation estimates are accounted for, the "UV" and "H $\alpha$ " SFRs agree within $10 \%$ across the entire range of galaxy masses.

6. We calibrate the relations that allow SFRs to be determined directly from $K$-corrected FUV and NUV magnitudes. They involve an FUV attenuation estimate from the UV color (equivalent to the UV slope) that we calibrated for normal star-forming galaxies, and find it to be less steep than the Meurer et al. (1999) relation. To obtain SFRs we find that one requires a conversion factor between the FUV luminosity and SFR that is 30\% lower than Kennicutt (1998) value. Such "simple" UV SFRs compare well with those obtained from the full SED fitting in the $0.1-10 M_{\odot} \mathrm{yr}^{-1}$ range, i.e., for normal star-forming galaxies.

7. For some $50 \%$ of the optically selected galaxies from SDSS, the emission lines are either too weak (red sequence galaxies), or they are contaminated by narrow-line AGNs, which precludes obtaining SFRs from $\mathrm{H} \alpha$ directly. In those cases B04 use calibrations between SFR and optical colors from SF galaxies and apply them to other classes of galaxies. We find that such calibrations are not reliable and lead to large overestimation of SFRs. UV, on the other hand, provides more secure SFR estimates.

8. We present stellar-mass dependent star formation histories for various classes of optically selected SDSS galaxies, from the starbursting dwarf galaxies with specific SFRs (SFR/ $M_{*}$ ) of $10^{-8} \mathrm{yr}^{-1}$ to essentially dead, red-sequence galaxies with 6 orders of magnitude lower specific SFR. We also present a volumecorrected version of these relationships, which give a better account of the SF at low masses. We quantify these relations in order to facilitate comparison with similar results obtained at higher redshifts.

9. Star-forming galaxies with no AGN form a very well defined linear star-forming sequence in specific SFR vs. mass plot, with the intrinsic $1 \sigma$ width of $0.5 \mathrm{dex}$ in $\log \mathrm{SFR} / M_{*}$. The sequence is $65 \%$ wider at the low mass end $\left(\sim 10^{8} M_{\odot}\right)$ than at high masses, consistent with a more stochastic (bursty) SF history for gas-rich dwarfs. The star-forming sequence terminates at $3 \times 10^{11} M_{\odot}$.

10. Galaxies whose ionizing flux (as measured in $3^{\prime \prime}$ fiber) comes primarily from young stars (star formation), those for which there is a narrow-line AGN component (mostly Seyfert $2 \mathrm{~s}$ and LINERs), and those that are spectroscopically quiescent (no detection of $\mathrm{H} \alpha$ lines), all occupy distinct positions in the specific SFR vs. stellar mass diagram, with the AGN hosts lying in between SF galaxies with no AGN, and the quiescent galaxies. Star-forming galaxies with no AGNs do not occupy the intermediate regions of the specific SFR — those are occupied exclusively by galaxies with AGNs.

11. If we take all galaxies forming the AGN branch in the BPT diagram (again from fiber measurements) and divide them by their AGN strength (as indicated by [O III] $\lambda 5007 \AA$ luminosity), we find that strong AGNs represent the massive continuation of the SF sequence defined by galaxies with no AGNs. Strong AGNs have similar SFRs as the most massive galaxies with no SF, but are more massive, driving their specific SFRs to intermediate values. Weak AGNs have similar masses as strong AGNs, but fall at lower star formation rates relative to strong AGNs, with a tail toward very low star formation rates that extends well into the domain of the optically red galaxies with no $\mathrm{H} \alpha$. While AGNs appear to be a massive continuation of the blue, star-forming sequence, their optical colors would place most of them onto the red sequence. We suggest an evolutionary sequence from massive star-forming to quiescent galaxies via strong and weak AGNs that bridge them. This scenario supports a picture of AGN feedback suppressing star formation in galaxies situated in very massive 
dark matter halos, the so-called radio mode AGNs (Croton et al. 2006). Further study is needed.

12. While mostly inactive, some galaxies with no detectable $\mathrm{H} \alpha$ in their fiber measurements exhibit SFRs as high as $\sim 1 M_{\odot} \mathrm{yr}^{-1}$. The optical colors of these galaxies are predominantly red. We find that many (1/4) of such galaxies display disturbed light profiles, sometimes in the form of fading spiral arms with no current SF detectable in $\mathrm{H} \alpha$. In many cases we see ringlike structures. Some of these are likely poststarburst systems. We find that up to $1 \%$ of UV SFR density comes from galaxies with no $\mathrm{H} \alpha$ lines.

13. We determine total SFR density in the local universe $(z=0.1)$ of $1.828_{-0.039}^{+0.148} \times 10^{-2} h_{70} M_{\odot} \mathrm{yr}^{-1} \mathrm{Mpc}^{-3}$ (for Chabrier IMF), which is in good agreement with most recent estimates, yet has a smaller error (which includes random and most systematic errors).

14. A large fraction (95\%) of SF takes place in blue sequence galaxies (NUV $-r<4$ ). SFR density increases in galaxies of increasing mass, and peaks in galaxies with masses $\approx 4 \times 10^{10} M_{\odot}-$ close to the "transition" mass of Kauffmann et al. (2003a; i.e., the mass at which the mass functions of red [old] and blue galaxies intersect). Galaxies in the mass range $9.3<\log M_{*}<10.6 \mathrm{ac}-$ count for $1 / 2$ of the total SFR density.

We demonstrate the ability of GALEX UV observations of the galaxies in the local universe to constrain SFRs of a statistically large number of galaxies with a wide dynamic range of SF activity. Besides characterizing the galaxies in the local universe, this study provides a better reference point for high-redshift investigations. This and the forthcoming studies based on GALEX will continue to provide a better understanding of the galaxy evolution, specifically the origin of the galaxy bimodality, the interconnection between the SF and AGN activity, and the general star formation history of galaxies spanning a range from gas-rich dwarfs to massive ellipticals.

We thank Janice C. Lee for insightful comments and helpful discussions. We thank the referee for numerous valuable suggestions. We also thank Alessandro Boselli and Michael Blanton. GALEX (Galaxy Evolution Explorer) is a NASA Small Explorer, launched in 2003 April. We gratefully acknowledge NASA's support for construction, operation, and science analysis for the GALEX mission, developed in cooperation with the Centre National d'Etudes Spatiales of France and the Korean Ministry of Science and Technology. Funding for the Sloan Digital Sky Survey (SDSS) and SDSS-II has been provided by the Alfred P. Sloan Foundation, the Participating Institutions, the National Science Foundation, the U.S. Department of Energy, the National Aeronautics and Space Administration, the Japanese Monbukagakusho, and the Max Planck Society, and the Higher Education Funding Council for England. This research has made use of NASA's Astrophysics Data System.

Facilities: GALEX, Sloan
Abazajian, K., et al. 2004, AJ, 128, 502

Adelman-McCarthy, J. K., et al. 2006, ApJS, 162, 38

Baldry, I. K., Glazebrook, K., Brinkmann, J., Ivezić, Ž., Lupton, R. H., Nichol, R. C., \& Szalay, A. S. 2004, ApJ, 600, 681

Baldwin, J. A., Phillips, M. M., \& Terlevich, R. 1981, PASP, 93, 5

Bell, E. F., \& Kennicutt, R. C., Jr. 2001, ApJ, 548, 681

Bell, E. F., et al. 2004, ApJ, 608, 752

Bertin, E., \& Arnouts, S. 1996, A\&AS, 117, 393

Blanton, M. R., \& Roweis, S. 2007, AJ, 133, 734

Blanton, M. R., et al. 2005, AJ, 129, 2562

Boissier, S., et al. 2007, ApJS, 173, 524

Boselli, A., Gavazzi, G., Donas, J., \& Scodeggio, M. 2001, AJ, 121, 753

Brinchmann, J., Charlot, S., White, S. D. M., Tremonti, C., Kauffmann, G., Heckman, T., \& Brinkmann, J. 2004, MNRAS, 351, 1151 (B04)

Brown, T. M., Bowers, C. W., Kimble, R. A., Sweigart, A. V., \& Ferguson, H. C. 2000, ApJ, 532, 308

Bruzual, A., G., \& Charlot, S. 1993, ApJ, 405, 538

Bruzual, G., \& Charlot, S. 2003, MNRAS, 344, 1000

Buat, V., Boselli, A., Gavazzi, G., \& Bonfanti, C. 2002, A\&A, 383, 801

Buat, V., et al. 2005, ApJ, 619, L51

Calzetti, D., Kinney, A. L., \& Storchi-Bergmann, T. 1994, ApJ, 429, 582

Chabrier, G. 2003, PASP, 115, 763

Charlot, S., \& Fall, S. M. 2000, ApJ, 539, 718

Charlot, S., \& Longhetti, M. 2001, MNRAS, 323, 887

Chester, C., \& Roberts, M. S. 1964, AJ, 69, 635

Cortese, L., et al. 2006, ApJ, 637, 242

Cram, L., Hopkins, A., Mobasher, B., \& Rowan-Robinson, M. 1998, ApJ, 507, 155

Croton, D. J., et al. 2006, MNRAS, 365, 11

David, L. P., Jones, C., \& Forman, W. 1992, ApJ, 388, 82

de Jong, T., Klein, U., Wielebinski, R., \& Wunderlich, E. 1985, A\&A, 147, L6

Donas, J., et al. 2007, ApJS, 173, 597

Faber, S. M., et al. 2007, ApJ, 665, 265

Ferland, G. J. 1996, University of Kentucky Internal Report

Feulner, G., Hopp, U., \& Botzler, C. S. 2006, A\&A, 451, L13

Gavazzi, G., Pierini, D., \& Boselli, A. 1996, A\&A, 312, 397

Gavazzi, G., \& Scodeggio, M. 1996, A\&A, 312, L29

Gil de Paz, A., et al. 2007, ApJS, 173, 185

Hanish, D. J., et al. 2006, ApJ, 649, 150

Holmberg, E. 1965, Ark. Astron., 3, 387

Hopkins, A. M., Connolly, A. J., Haarsma, D. B., \& Cram, L. E. 2001, AJ, 122, 288

Hubble, E. P. 1926, ApJ, 64, 321

\section{REFERENCES}

Hubble, E. P. 1936, Yale University Press

Iglesias-Páramo, J., Boselli, A., Gavazzi, G., \& Zaccardo, A. 2004, A\&A, 421, 887

Johnson, B. D., et al. 2007, ApJS, 173, 392

Kauffmann, G., et al. 2003a, MNRAS, 341, 54

2003b, MNRAS, 346, 1055 2007, ApJS, 173, 357

Kaviraj, S., et al. 2007, ApJS, 173, 619

Kennicutt, R. 1983, A\&A, 120, 219

Kennicutt, R. C., Jr. 1998, ARA\&A, 36, 189

Kilgard, R. E., Kaaret, P., Krauss, M. I., Prestwich, A. H., Raley, M. T., \& Zezas, A. 2002, ApJ, 573, 138

Kroupa, P. 2001, MNRAS, 322, 231

Larson, R. B., \& Tinsley, B. M. 1978, ApJ, 219, 46

Leonard, T., \& Hsu, J. S. J. 2003, Bayesian Methods: An Analysis for Statisticians and Interdisciplinary Researchers (Cambridge: Cambridge Univ. Press)

MacKay, D. J. C. 2003, Information Theory, Inference, and Learning Algorithms, Cambridge University Press

Madau, P., Ferguson, H. C., Dickinson, M. E., Giavalisco, M., Steidel, C. C., \& Fruchter, A. 1996, MNRAS, 283, 1388

Martin, D. C., et al. 2005, ApJ, 619, L1

2007, ApJS, 173, 342

Meurer, G. R., Heckman, T. M., \& Calzetti, D. 1999, ApJ, 521, 64

Milliard, B., Donas, J., Laget, M., Armand, C., \& Vuillemin, A. 1992, A\&A, 257, 24

Morrissey, P., et al. 2005, ApJ, 619, L7

2007, ApJS, 173, 682

Noeske, K. G., et al. 2007, ApJ, 660, L43

Panuzzo, P., Granato, G. L., Buat, V., Inoue, A. K., Silva, L., Iglesias-Paramo, J., \& Bressan, A. 2007, MNRAS, 375, 640

Papovich, C., et al. 2006, ApJ, 640, 92

Poggianti, B. M., \& Barbaro, G. 1997, A\&A, 325, 1025

Poggianti, B. M., Smail, I., Dressler, A., Couch, W. J., Barger, A. J., Butcher, H., Ellis, R. S., \& Oemler, A. J. 1999, ApJ, 518, 576

Rich, R. M., et al. 2005, ApJ, 619, L107

Rosa-González, D., Terlevich, E., \& Terlevich, R. 2002, MNRAS, 332, 283

Roussel, H., Sauvage, M., Vigroux, L., \& Bosma, A. 2001, A\&A, 372, 427

Salim, S., et al. 2005, ApJ, 619, L39

Salpeter, E. E. 1955, ApJ, 121, 161

Sandage, A. 1972, ApJ, 176, 21

Schawinski, K., et al. 2007, ApJS, 173, 512

Schechter, P. 1976, ApJ, 203, 297

Schiminovich, D., et al. 2005, ApJ, 619, L47 
Scranton, R., Connolly, A. J., Szalay, A. S., Lupton, R. H., Johnston, D., Budavari, T., Brinkman, J., \& Fukugita, M. 2005, AJ, submitted (astro-ph/0508564)

Searle, L., Sargent, W. L. W., \& Bagnuolo, W. G. 1973, ApJ, 179, 427

Seibert, M., et al. 2005, ApJ, 619, L55

Skrutskie, M. F., et al. 2006, AJ, 131, 1163

Springel, V., Di Matteo, T., \& Hernquist, L. 2005, ApJ, 620, L79

Strauss, M. A., et al. 2002, AJ, 124, 1810

Sullivan, M., Mobasher, B., Chan, B., Cram, L., Ellis, R., Treyer, M., \& Hopkins, A. 2001, ApJ, 558, 72
Sullivan, M., Treyer, M. A., Ellis, R. S., Bridges, T. J., Milliard, B., \& Donas, J. 2000, MNRAS, 312, 442

Trammell, G. B., Vanden Berk, D. E., Schneider, D. P., Richards, G. T., Hall, P. B., Anderson, S. F., \& Brinkmann, J. 2007, AJ, 133, 1780

van den Bergh, S. 1976, ApJ, 206, 883

Wang, B., \& Heckman, T. M. 1996, ApJ, 457, 645

Wyder, T. K., et al. 2007, ApJS, 173, 293

Yi, S. K., et al. 2005, ApJ, 619, L111 